\title{
Aging Increases Susceptibility to High Fat Diet-Induced Metabolic Syndrome in C57BL/6 Mice: Improvement in Glycemic and Lipid Profile after Antioxidant Therapy
}

\author{
Valéria Nunes-Souza, ${ }^{1,2,3}$ Cheila Juliana César-Gomes, ${ }^{1,2}$ Lucas José Sá Da Fonseca, ${ }^{1,2}$ \\ Glaucevane Da Silva Guedes, ${ }^{1,2,4}$ Salete Smaniotto, ${ }^{5}$ and Luíza Antas Rabelo ${ }^{1,2,6}$ \\ ${ }^{1}$ Laboratório de Reatividade Cardiovascular (LRC), Núcleo de Síndrome Metabólica, \\ Instituto de Ciências Biológicas e da Saúde (ICBS), Universidade Federal de Alagoas (UFAL), \\ Avenida Lourival Melo Mota, s/n, Cidade Universitária, 57072-900 Maceió, AL, Brazil \\ ${ }^{2}$ National Institute of Science and Technology in Nano-Biopharmaceutics (N-BIOFAR), Avenida Antônio Carlos, \\ s/n, Pampulha, 31270-901 Belo Horizonte, MG, Brazil \\ ${ }^{3}$ Departamento de Fisiologia e Farmacologia, Centro de Ciências Biológicas (CCB), Universidade Federal de Pernambuco (UFPE), \\ Avenida Professor Moraes Rego 1235, Cidade Universitária, 50670-901 Recife, PE, Brazil \\ ${ }^{4}$ Faculdade de Nutrição (FANUT), Universidade Federal de Alagoas (UFAL), Avenida Lourival Melo Mota, s/n, \\ Cidade Universitária, 57072-900 Maceió, AL, Brazil \\ ${ }^{5}$ Laboratório de Biologia Celular, Instituto de Ciências Biológicas e da Saúde (ICBS), Universidade Federal de Alagoas (UFAL), \\ Avenida Lourival Melo Mota, s/n, Cidade Universitária, 57072-900 Maceió, AL, Brazil \\ ${ }^{6}$ Max Delbrück Center for Molecular Medicine, Robert-Rössle-Straße 10, 13125 Berlin, Germany
}

Correspondence should be addressed to Luíza Antas Rabelo; luizaa.rabelo@gmail.com

Received 30 December 2015; Accepted 18 January 2016

Academic Editor: Vladimir Jakovljevic

Copyright (C) 2016 Valéria Nunes-Souza et al. This is an open access article distributed under the Creative Commons Attribution License, which permits unrestricted use, distribution, and reproduction in any medium, provided the original work is properly cited.

Nonalcoholic fatty liver disease (NAFLD) has been considered a novel component of the metabolic syndrome (MetS), with the oxidative stress participating in its progression. This study aimed to evaluate the metabolic profile in young and old mice with MetS, and the effects of apocynin and tempol on glycemic and lipid parameters. Young and old C57BL/6 mice with high fat diet(HFD-) induced MetS received apocynin and tempol $50 \mathrm{mg} \cdot \mathrm{kg}^{-1} / \mathrm{day}$ in their drinking water for 10 weeks. After HFD, the young group showed elevated fasting glucose, worsened lipid profile in plasma, steatosis, and hepatic lipid peroxidation. Nevertheless, the old group presented significant increase in fasting insulin levels, insulin resistance, plasma and hepatic lipid peroxidation, and pronounced steatosis. The hepatic superoxide dismutase and catalase activity did not differ between the groups. Tempol and apocynin seemed to prevent hepatic lipid deposition in both groups. Furthermore, apocynin improved glucose tolerance and insulin sensitivity in old mice. In summary, old mice are more susceptible to HFD-induced metabolic changes than their young counterparts. Also, the antioxidant therapy improved insulin sensitivity and glucose tolerance, and in addition, apocynin seemed to prevent the HFD-induced hepatic fat deposition, suggesting an important role of oxidative stress in the induction of NAFLD.

\section{Introduction}

Nonalcoholic fatty liver disease (NAFLD), characterized by fatty infiltration of the liver, has been considered a novel component of the metabolic syndrome (MetS) [1-4], the latter being a multifactorial condition that gathers various cardiovascular risk factors and metabolic disorders $[2,3]$.

NAFLD encompasses various degrees of liver damage, ranging from simple steatosis to a complex and more dangerous presentation, steatohepatitis [5], and it is often 
accompanied by oxidative stress [6], a condition of imbalance in the prooxidant/antioxidant balance in favor of prooxidants [7].

The liver is a central organ for the maintenance of systemic lipid and glucose homeostasis and, due to this fact, it is susceptible to oxidative stress damage $[8,9]$. The latter plays a critical role in the progression of metabolic disorders, including insulin resistance, one of the conditions which characterize the NAFLD [4]. In addition, oxidative stress may induce several pathophysiological events in the liver, such as detrimental effects on hepatocytes by damaging DNA, lipids, and proteins, ultimately leading to disruptions in cellular homeostasis $[2,10]$.

Nicotinamide adenine dinucleotide phosphate oxidase $(\mathrm{NAD}(\mathrm{P}) \mathrm{H}$ oxidase) is an enzyme involved in the progression of the disorders related to the MetS, such as liver injury [11], hyperglycemia [12], increased oxidative stress in diabetes and obesity [2, 13], hypertension [14], and impaired endotheliumdependent dilation [15]. Also, that enzyme appears to play an essential role in a higher insulin-stimulated hydrogen peroxide $\left(\mathrm{H}_{2} \mathrm{O}_{2}\right)$ generation in skeletal muscle [16].

Although NAD $(\mathrm{P}) \mathrm{H}$ oxidase is the major source of reactive oxygen species (ROS) production $[13,17]$, the antioxidant enzymes superoxide dismutase (SOD), catalase (CAT), and glutathione peroxidase (GPx) have a critical importance in the metabolism of ROS [7]. SOD is responsible for dismuting the superoxide anion $\left({ }^{\circ} \mathrm{O}_{2}{ }^{-}\right)$into $\mathrm{H}_{2} \mathrm{O}_{2}$, which, in turn, is removed by CAT and GPx, finally being converted into water and $\mathrm{O}_{2}$ [7]. In this scenario, these enzymes exert an important protective function against ROS, which are increased with age $[18,19]$ and in high fat diet- (HFD-) induced obesity, as well as in MetS and NAFLD animal models $[1,4,9,20]$.

Even though several studies have been conducted to assess the metabolic effects of the consumption of HFD in animal models $[16,21,22]$, little is known about its effect concomitant with antioxidant therapy in old age and in the MetS. Based upon this scenario, we hypothesized that aged mice are more sensitive to HFD-induced metabolic changes than younger mice and that the concomitant antioxidant therapy is effective in reducing the negative metabolic impacts from HFD consumption. In order to test this hypothesis, we assessed the metabolic profile in young and old C57BL6 mice presenting with MetS and sought to investigate the preventive effects of apocynin, a NAD $(\mathrm{P}) \mathrm{H}$ oxidase inhibitor, and tempol, a SOD mimetic, on HFD-induced metabolic changes in those animals.

\section{Materials and Methods}

2.1. Animals and Experimental Procedures. C57BL/6 male young and old mice used in this study were purchased from Federal University of Alagoas at 6-8 weeks and 54-56 weeks of age, respectively. During all the experimental period, mice were kept on a 12-hour light/dark cycle and fed and hydrated ad libitum, with controlled humidity and temperature. All procedures were carried out in accordance with the criteria outlined in the Guide for the Care and Use of Laboratory Animals (NIH publication 86-23, 1996) and approved by the
Institutional Ethics Committee of the Federal University of Alagoas (Protocol 005208/2010-46).

Young $(\mathrm{Y})$ and old $(\mathrm{O})$ mice were divided into two groups and fed different diets during 14 weeks: high fat diet, which was developed by our group, with the other nutrient contents in accordance with the American Institute of Nutrition's recommendation (AIN-93) [23, 24] (HF-Y and HF-O) $(58.4 \%$ kcal lipids from lard; $26.6 \%$ kcal carbohydrates, i.e., sucrose; $15 \% \mathrm{kcal}$ protein, i.e., casein) or standard chow diet (CT-Y and CT-O) (11.8\% kcal lipids, $62.6 \% \mathrm{kcal}$ carbohydrates, and $25.6 \% \mathrm{kcal}$ protein). After 4 weeks of HFD (the required period for the glycemic alterations to appear, a time interval previously detected in other experimental groups conducted by our laboratory, with blood glucose levels assessed weekly-Accu-Chek Performa, Roche ${ }^{\circledast}$, São Paulo, Brazil), the HF-Y and HF-O groups were divided into three subgroups according to their treatment: (1) positive control that continued receiving only HFD and normal water (HF-Y and HF-O); (2) HFD plus oral treatment with tempol (50 mg. $\mathrm{kg}^{-1} / \mathrm{day}$, dissolved in drinking water) (HFT$\mathrm{Y}$ and HFT-O); (3) HFD plus oral treatment with apocynin (50 mg. $\mathrm{kg}^{-1} /$ day, dissolved in drinking water) (HFA-Y and HFA-O). The exact duration of the tempol and apocynin interventions was 10 weeks. The dosages of tempol and apocynin were recalculated weekly, taking into account the mean body weight recorded every week and the liquid consumption of the groups.

\subsection{Intraperitoneal Glucose Tolerance Test (GTT) and Insulin} Tolerance Test (ITT). At the end of treatment, fasting blood glucose was assessed ( $12 \mathrm{~h}$ fast) using a glucometer (AccuChek Performa, Roche, São Paulo, Brazil). Next, 2 g glucose/kg of body weight was intraperitoneally injected and blood from the tail vein was collected again at 15, 30, 60, 90 , and 120 minutes for measuring glucose levels. Two days later, baseline blood glucose was measured in fed state. Sequentially, insulin ( 0.75 units/kg of body weight; Humulin ${ }^{\circledR}$, Eli Lilly, Indianapolis, IN) was intraperitoneally administered and tail blood samples were obtained at 15, 30, and 60 minutes to obtain blood glucose levels.

2.3. Euthanasia and Organ Collection. After intraperitoneal a nesthesia using a xylazine/ketamine solution $\left(10 / 110 \mathrm{mg} \cdot \mathrm{kg}^{-1}\right)$, the cephalocaudal length and abdominal circumference were measured $(\mathrm{cm})$ and the animals were sacrificed by exsanguination through cardiac puncture of the right ventricle. Blood was collected and centrifuged and plasma was separated and stored at $-80^{\circ} \mathrm{C}$ until biochemical analyses were performed. Liver, adipose tissues, heart, gastrocnemius muscle, and intestine were carefully perfused, removed, and weighed. Next, one piece of the right hepatic lobe was selected for histological analysis, and two more pieces ( $100 \mathrm{mg}$ ) were immediately snap-frozen in dry ice and stored at $-80^{\circ} \mathrm{C}$ for assessing lipid profile and the activities of antioxidant enzymes. Similarly, adipose tissues (epididymal, perirenal, and mesenteric) were stored at $-80^{\circ} \mathrm{C}$ for further analysis. White adipose tissue (WAT) index was calculated following the formula: WAT index $(\%)=[$ (epididymal fat $(\mathrm{g})+$ perirenal fat $(\mathrm{g})) /($ body weight $(\mathrm{g})) * 100$ ] [25]. The 
relative weight (\%) of adipose tissue was calculated as follows: relative weight $(\%)=[($ adipose tissue $(\mathrm{g}) /$ body weight $(\mathrm{g}))$ * 100]. Liver, gastrocnemius, heart, and intestine mass were normalized by tibial length and expressed as $\mathrm{g} / \mathrm{mm}$ of tibial length. Finally, right tibias were also removed and had their lengths measured.

2.4. Plasma Lipid Analysis. Total cholesterol, triglyceride, and free fatty acids (FFA) levels in plasma were assayed using commercial kits (enzymatic colorimetric assay; Labtest ${ }^{\oplus}$, Lagoa Santa, Brazil, and Wako Chemicals $\mathrm{GmbH}^{\oplus}$, Neuss, Germany), following the manufacturer's instructions with adaptations for microplate (Thermo Scientific ${ }^{\oplus}$, Software 2.4 Multiskan Spectrum, Vantaa, Finland). VLDL was calculated as follows: $\mathrm{VLDL}=$ triglyceride/5 [26].

2.5. Insulin Measurement and Markers of Insulin Resistance. Insulin levels were quantified using an ELISA assay (Millipore ${ }^{\circledast}$, Schwalbach, Germany) according to the manufacturer's instructions. The product of fasting triglycerides and glucose (TyG index) was calculated following the formula: TyG index $=$ Ln [fasting triglyceride $\left(\mathrm{mg} \cdot \mathrm{dL}^{-1}\right) \times$ fasting glucose $\left.\left(\mathrm{mg} \cdot \mathrm{dL}^{-1}\right) / 2\right][27,28]$. The homeostasis model assessment (HOMA index) was calculated as follows: HOMA index $=\left[\right.$ fasting glucose $\left(\mathrm{mg} \cdot \mathrm{dL}^{-1}\right) \times$ fasting insulin $\left(\mu \mathrm{U} \cdot \mathrm{mL}^{-1}\right) /$ 405] [29].

2.6. Evaluation of Liver Injury. Commercial kits (Labtest, Lagoa Santa, Brazil) adapted for microplate reading (Thermo Scientific, Software 2.4 Multiskan Spectrum, Vantaa, Finland) were used for evaluating in plasma the degree of liver injury and function by measuring the enzymatic concentrations of alanine aminotransferase (ALT), aspartate aminotransferase (AST), alkaline phosphatase (ALP), $\gamma$-glutamyltransferase $(\gamma-\mathrm{GT})$, and lactate dehydrogenase (LDH). Levels of total protein [30] and albumin were also determined.

2.7. Liver Histological Analysis. In order to investigate liver structure and the occurrence of steatosis, small hepatic fragments were fixed in $10 \%$ buffered formaldehyde, embedded in paraffin, sectioned at 4-6 $\mu \mathrm{m}$, and stained with hematoxylin and eosin (H\&E). The sections from each animal were histologically examined in light microscope (Olympus ${ }^{\circledast}$, Tokyo, Japan) and photographed (Kodak digital camera, Eastman Kodak Company, Rochester, NY, USA).

2.8. Liver Lipids Analysis. Total hepatic lipids were extracted from liver samples according to a gravimetric standard method [31], followed by measurement of cholesterol and triglyceride levels using commercial kits (enzymatic colorimetric assays; Labtest, Lagoa Santa, Brazil) according to the manufacturer's instructions, with adaptations for microplate (Thermo Scientific, Software 2.4 Multiskan Spectrum, Vantaa, Finland), and VLDL as described [26].

2.9. Protein Extraction from Liver Samples. From each mouse, a piece of frozen liver was homogenized in a RIPA lysis buffer (Cell Signaling ${ }^{\oplus}$, Beverly, MA, USA) plus protease and phosphatase inhibitor cocktails (Roche, Mannheim, Germany).
Following the extraction, total protein concentration was quantified by the Bradford method [30] and the samples were stored at $-80^{\circ} \mathrm{C}$ for subsequent measurements.

2.10. Measurement of Lipid Peroxidation. Thiobarbituric acid reactive substances (TBARS), mainly malondialdehyde (MDA), are widely used as a marker of lipid peroxidation. Such quantification was performed according to Ohkawa et al. [32] with some adaptations. Briefly, $100 \mu \mathrm{L}$ of hepatic homogenate samples or $100 \mu \mathrm{L}$ of plasma was individually mixed with $10 \mu \mathrm{L}$ of butylated hydroxytoluene (final concentration = $\left.1 \mathrm{mmol} \cdot \mathrm{L}^{-1}\right)$ and $1250 \mu \mathrm{L}$ of thiobarbituric acid (1.3\%; Sigma Aldrich $^{\oplus}$, Seelze, Germany). Then, samples were heated at $100^{\circ} \mathrm{C}$ during 60 minutes, being next incubated in ice bath $(\sim$ $4^{\circ} \mathrm{C}$ ) for 10 minutes to stop the reaction. Sequentially, samples were centrifuged at $1600 \mathrm{~g}$ per 15 minutes at $4^{\circ} \mathrm{C}$. In order to remove the interfering absorbance, for each sample, the value of the absorbance at $600 \mathrm{~nm}$ was subtracted from the value read at $532 \mathrm{~nm}$. Finally, values were normalized for total protein concentration and expressed as $\mathrm{nM} \cdot \mathrm{mg}$ protein $^{-1}$ [30].

2.11. Superoxide Dismutase (SOD) and Catalase (CAT) Activities. Total SOD activity was assessed in $20 \mu \mathrm{L}$ of liver homogenate with a commercial colorimetric kit (Sigma Aldrich, Seelze, Germany) following the manufacturer's instructions and read in a microplate reader (Thermo Scientific, Software 2.4 Multiskan Spectrum, Vantaa, Finland) at $450 \mathrm{~nm}$. The obtained values were normalized by total protein concentration in the liver [30] and expressed as IU.protein ${ }^{-1}$, mg of hepatic protein. Catalase activity was measured according to Xu et al. [33], being expressed as $\mu \mathrm{mol} / \mathrm{min} / \mathrm{mL} \cdot$ protein $^{-1}, \mathrm{mg}$ [30].

2.12. Statistical Analysis. The data are expressed as mean \pm standard error of the mean (SEM), and $p<0.05$ was considered statistically significant. Analyses were performed using the one- or two-way ANOVA, when applicable (followed by Bonferroni’s post hoc test) (Graph pad Prism ${ }^{\circledR}$ 5.0, San Diego, CA, USA).

\section{Results}

3.1. General Parameters, Fat Deposition, and Lipid Profile in Mice with MetS and Treated with Antioxidant Therapy. The measurements of food intake, animal growth, abdominal circumference, and lipids and glucose metabolism, variables which can be changed during the consumption of a HFD, were done to evaluate the possible effects of HFD and of the antioxidant treatment with apocynin and tempol in mice at different ages.

The data showed that the food intake, regardless of age, was statistically lower in HFD-fed groups (HF-Y and HF$\mathrm{O}$; Table 1). However, the energy consumed did not differ between groups, since the HFD displays a caloric content greater than the chow diet, thus normalizing the caloric intake (data not shown). Besides, abdominal circumference was increased in HF-O compared to CT, without differences between the young groups. With respect to the influence of 


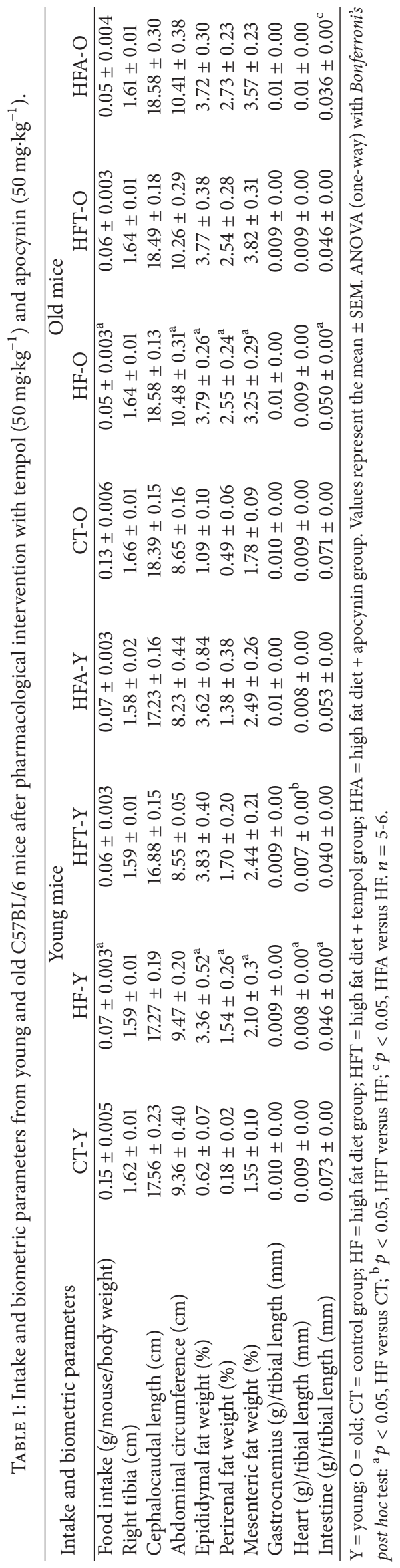




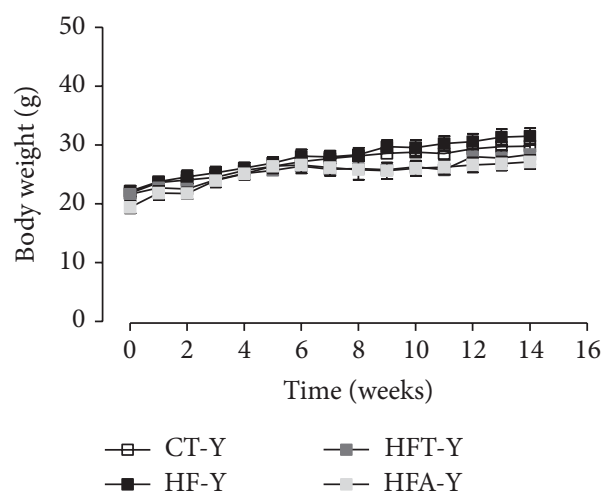

(a)

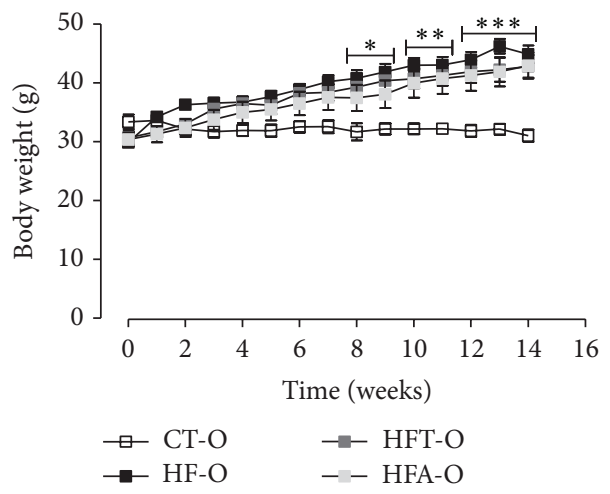

(c)
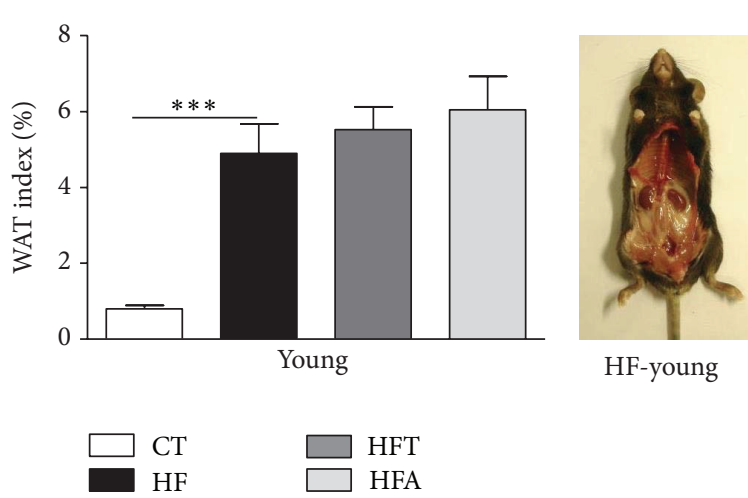

(b)

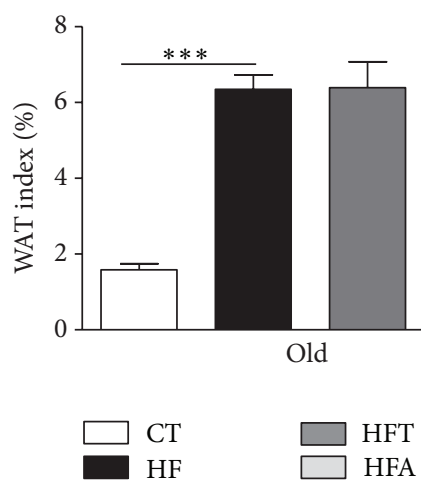

(d)

FIGURE 1: Body weight and WAT index. Monitoring of body weight $(\mathrm{g})$ during the weeks before and after pharmacological intervention with tempol $\left(50 \mathrm{mg} \cdot \mathrm{kg}^{-1}\right)$ and apocynin $\left(50 \mathrm{mg} \cdot \mathrm{kg}^{-1}\right)$ in young (a) and old (c) C57BL/6 mice. WAT index (\%) and representative pictures of the $\mathrm{HF}$ diet animals after pharmacological intervention in young (b) and old (d) C57BL/6 mice. CT = control group; HF = high fat diet group; HFT $=$ high fat diet + tempol group; HFA $=$ high fat diet + apocynin group. Each point of the graph represents the mean \pm SEM. ANOVA (two-way) with Bonferroni's post hoc test: ${ }^{*} p<0.05 ;{ }^{* *} p<0.01 ;{ }^{* * *} p<0.001$, CT versus HF. Each bar graph represents the mean \pm SEM. ANOVA (one-way) with Bonferroni's post hoc test: ${ }^{* * *} p<0.001$, CT versus $\mathrm{HF}(n=5-6)$.

HFD on animal growth, no statistical difference was observed in tibial and cephalocaudal length between young and old groups. Neither tempol nor apocynin had effect on any of these aforementioned parameters during HFD intake in both groups (Table 1).

Although no significant difference in body weight was observed between the young groups during the HFD consumption (Figure 1(a)), the WAT index (Figure 1(b)) and relative epididymal, perirenal, and mesenteric fat weight (Table 1) were significantly higher. However, in one of the old groups (HF-O), a significant increase was observed in body weight compared with CT-O from the 8th week $(p<0.05)$ until the end of treatment $(p<0.001)$ (Figure 1(c)), indicating that aging enhances the metabolic effects associated with HFD consumption. The WAT index (Figure 1(d)) and the relative epididymal, perirenal, and mesenteric fat weight (Table 1) were also significantly higher in old animals after HFD consumption. In both young and old groups, apocynin and tempol had no effects on body weight, WAT index, and relative adipose tissue weight during HFD intake when compared to the respective control (HF-Y and HF-O, resp.).

The HFD did not change skeletal muscle mass (gastrocnemius mass) in young and old groups (Table 1). On the other hand, the intestinal mass was significantly decreased in both young and old groups that received HFD. In addition, the heart mass was significantly lower in the HF-Y but not in HF-O (Table 1), suggesting that induction of muscle loss by consumption of HFD is more pronounced in the younger compared to older age. HF-Y mice, although not presenting an increase in body weight, showed a markedly elevated concentration of total cholesterol, triglycerides, VLDL, and FFA in plasma compared with CT-Y (Figures 2(a), 2(c), 2(e), and $2(\mathrm{~g})$, resp.). Similarly, HF-O mice presented elevated total cholesterol, triglycerides, and VLDL levels but not FFA compared to the CT-O (Figures 2(b), 2(d), 2(f), and 2(h), resp.). Lipid peroxidation was not different between the young groups, but the old HFD group presented significant increase in this parameter, which was decreased after apocynin treatment (Figures 2(i) and 2(j)). In the old group, both tempol and apocynin were also able to decrease the levels of triglycerides and VLDL in plasma (Figures 2(d) and 2(f)).

3.2. Glycemic Profile in Mice with MetS and Treated with Antioxidant Therapy. As shown in Figure 3, the basal levels of glucose (Time 0 ) in young and old mice were not different. However, after receiving HFD during 4 weeks (Time 1), 

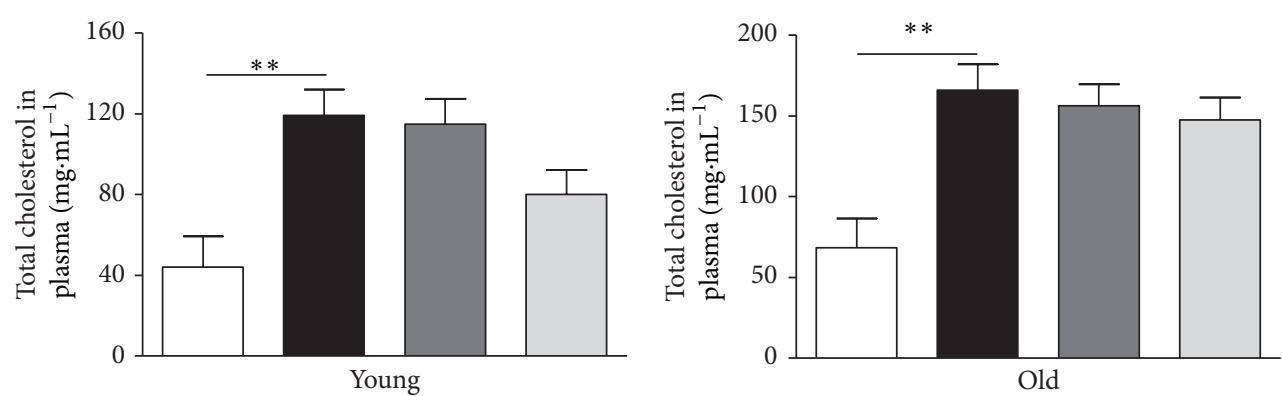

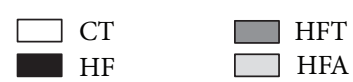

(a)
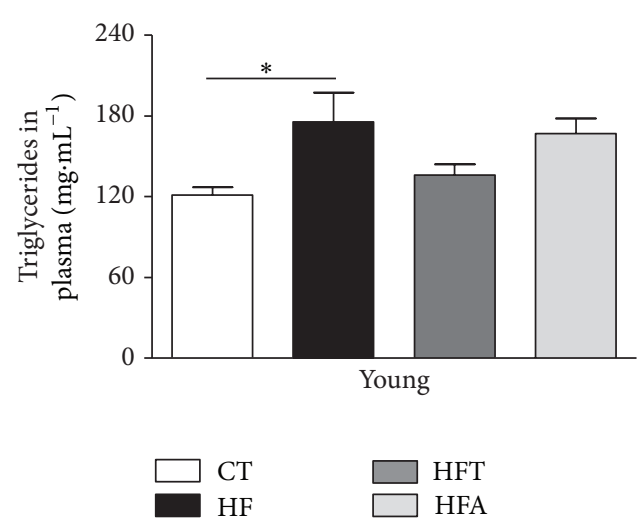

(c)
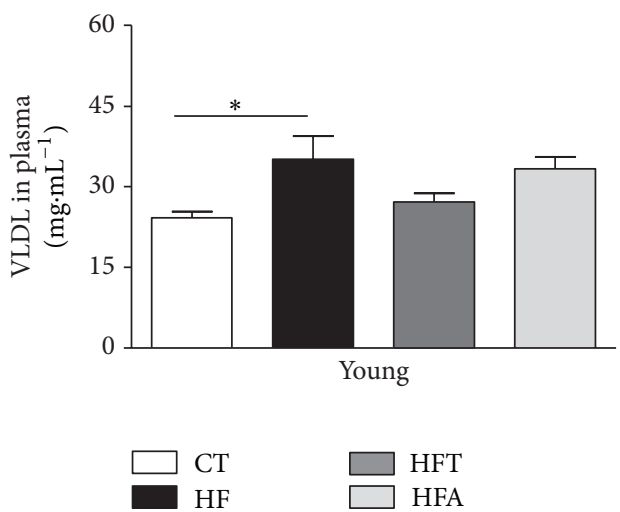

(e)

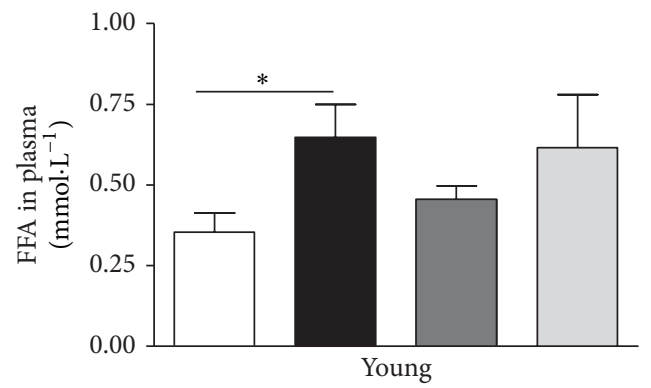

$\square$ CT
HF

(g)

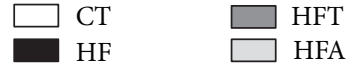

(b)
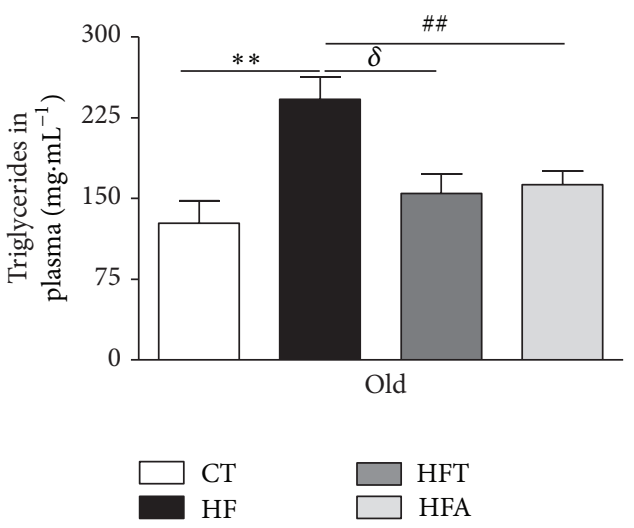

(d)
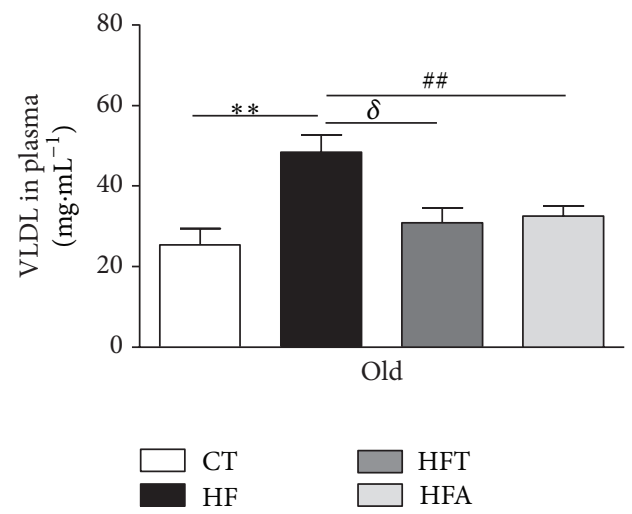

(f)
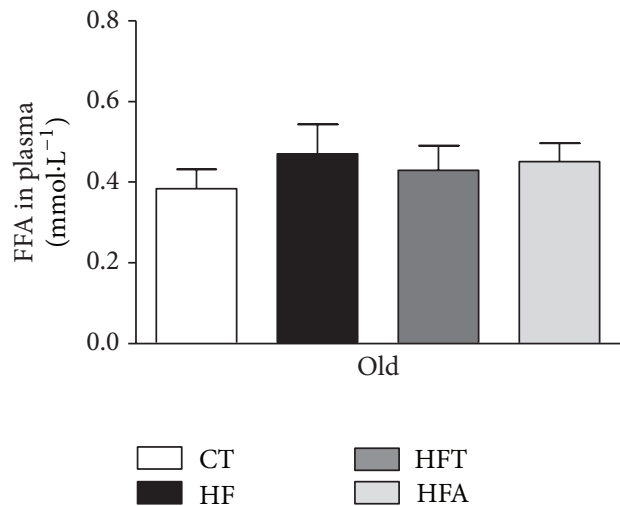

(h)

Figure 2: Continued. 

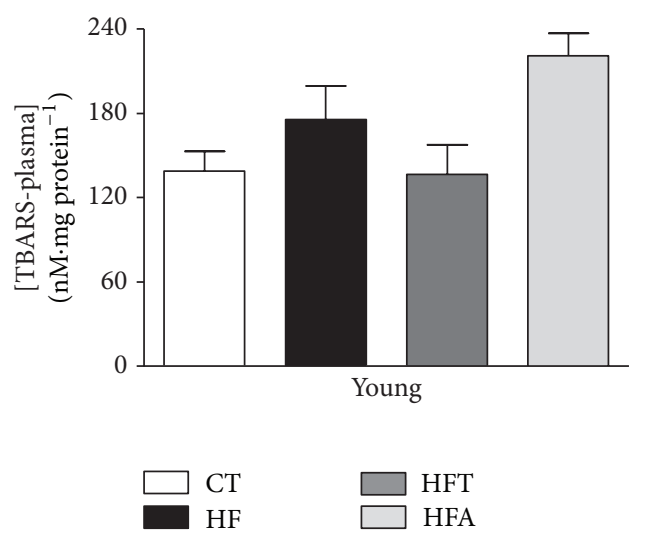

(i)
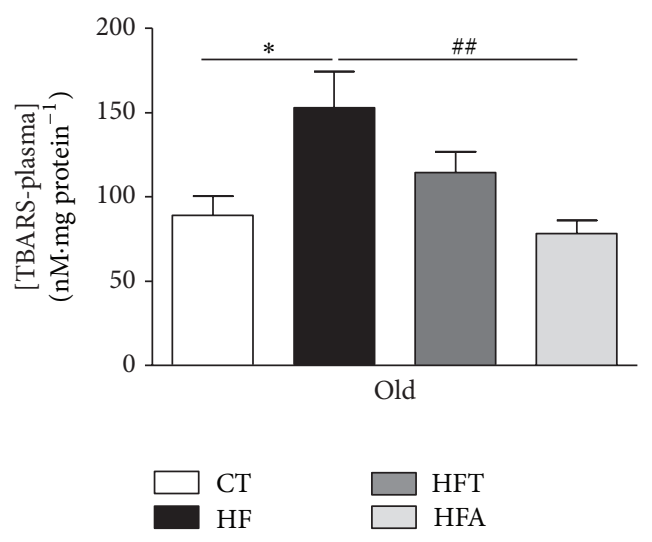

(j)

FIgURE 2: Lipid profile in plasma. Total cholesterol in plasma ( $\mathrm{mg}^{-\mathrm{mL}^{-1}}$ ) in young (a) and old (b) C57BL/6 mice. Triglycerides in plasma $\left(\mathrm{mg} \cdot \mathrm{mL}^{-1}\right)$ in young $(\mathrm{c})$ and old (d) C57BL/6 mice. Very-low-density lipoprotein (VLDL) in plasma (mg. $\left.\mathrm{mL}^{-1}\right)$ in young (e) and old (f) C57BL/6 mice. Free fatty acids (FFA) in plasma $\left(\mathrm{mmol} \cdot \mathrm{L}^{-1}\right)$ in young $(\mathrm{g})$ and old $(\mathrm{h}) \mathrm{C} 57 \mathrm{BL} / 6$ mice. Lipid peroxidation in plasma ([TBARS] $\mathrm{nM} \cdot \mathrm{mg}_{\text {protein }}{ }^{-1}$ ) in young (i) and old (j) C57BL/6 mice. CT $=$ control group; HF = high fat diet group; HFT = high fat diet + tempol group; HFA = high fat diet + apocynin group. Each bar graph represents the mean \pm SEM. ANOVA (one-way) with Bonferroni's post hoc test: ${ }^{*} p<0.05 ;{ }^{* *} p<0.01, \mathrm{CT}$ versus HF; ${ }^{\delta} p<0.05$, HFT versus HF; ${ }^{\# \#} p<0.01$, HFA versus $\mathrm{HF}(n=5-6)$.

these groups (HF-Y and HF-O) presented significantly higher fasting glucose compared to their respective CT groups (CT$\mathrm{Y}$ and CT-O). At this point, we started the pharmacological intervention with tempol and apocynin during 10 weeks. At the end of treatment (Time 2), regardless of age, no statistical difference in glucose concentration was observed for tempol groups (HFT-Y, HFT-O) compared to their respective HFD groups (HF-Y and HF-O). Nevertheless, apocynin significantly decreased the final fasting glucose in old animals with MetS, but not in the young ones (Figures 3(a) and 3(b)).

HF-Y and HF-O mice presented increased glucose intolerance (Figures 3(c) and 3(d)). This effect was evident by the higher time-dependent glucose concentration at 15, 30, 60, and $90 \mathrm{~min}$ after the intraperitoneal injection of glucose, as well as by the significant increase in the AUC in the HF$\mathrm{Y}$ and HF-O groups compared with the correspondent CT group. Apocynin, a $\mathrm{NAD}(\mathrm{P}) \mathrm{H}$ oxidase inhibitor, seems to prevent the occurrence of such effects in both young and old mice with MetS. On the other hand, tempol, a SOD mimetic, was shown to be capable of reducing the occurrence of the aforementioned changes only in old mice.

Interestingly, the young mice (HF-Y) did not show decreased insulin sensitivity after HFD consumption, a fact observed in old mice (HF-O) during the ITT, and when calculating the AUC from these data (Figures 3(e) and 3(f)). Time-dependent glucose concentration in ITT and the AUC decreased in the HFT-O and HFA-O compared with the HFO group, indicating that both apocynin and tempol improved insulin sensitivity in old mice. In addition, we measured the fasting insulin concentrations in plasma, with no differences observed in young groups after HFD, but with a significant increase in the old group. Additionally, in the treated groups, neither tempol nor apocynin had effects on that parameter in the old group. However, in the young group, both treatments decreased the insulin levels compared to the HFD group
(Table 2). Analysis of the insulin resistance markers, TyG index and HOMA index, showed that only in old animals these parameters were increased after HFD consumption, with both treatments, tempol and apocynin, being able to significantly decrease the TyG index in old animals (Table 2).

3.3. Function, Lipid Profile, and Redox State in Liver Samples of Mice with MetS and Treated with Antioxidant Therapy. In order to evaluate the effect of the improved glycemic profile on liver metabolism after pharmacological intervention, we assessed markers of liver injury and function, fat accumulation, and hepatic redox balance.

The liver weight in young mice that consumed HFD was significantly lower compared to that observed in animals fed standard chow diet (Figure 4(a)). On the other hand, the HFO showed a significant increase in liver weight (Figure 4(b)). The analysis of plasma markers of hepatocellular injury (ALT, AST, and LDH), canalicular enzymes (ALP, $\gamma$-GT), and markers of liver function (albumin and total proteins) is shown in Table 2 . In both young and old mice, AST and ALT levels were increased in HF group compared to CT group, indicating the elevated hepatic injury in these animals after the HFD consumption. The treatment with tempol decreased the levels of AST and ALT in plasma of young animals, but not in the old ones. Additionally, the other hepatic markers evaluated were not found to be different between the groups.

The quantification of total lipids and fractions (total cholesterol, triglycerides, and VLDL) showed an increase after HFD consumption in both young and old animals (Figures $4(\mathrm{c})-4(\mathrm{j})$ ). No significant changes were observed in the concentration of all the parameters listed above in groups treated with tempol. However, apocynin treatment was able to decrease the levels of triglycerides and VLDL in liver of young animals with MetS (Figures 4(e) and 4(f)). 

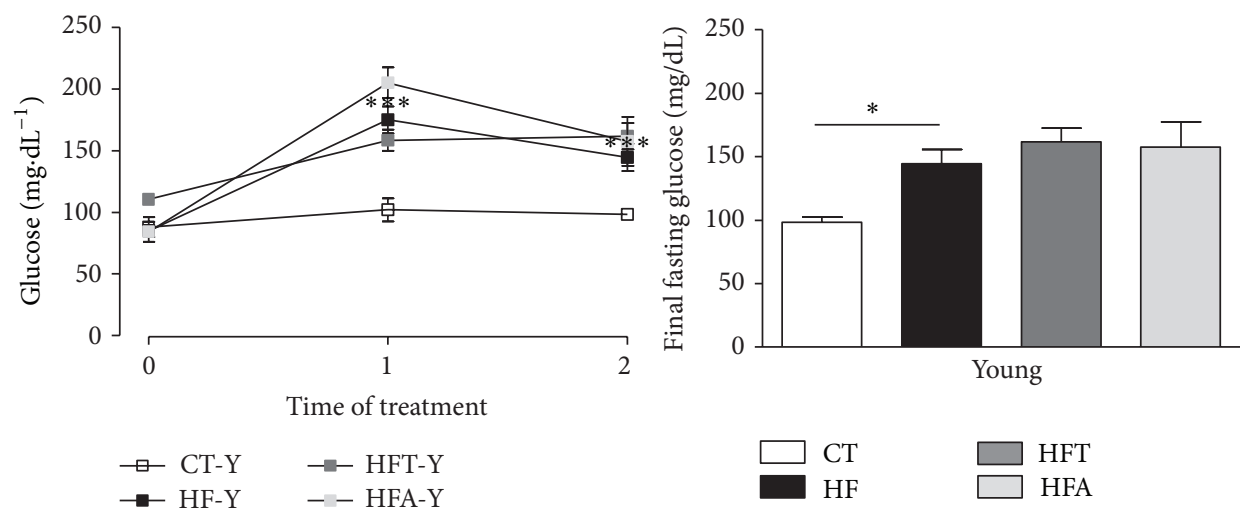

(a)
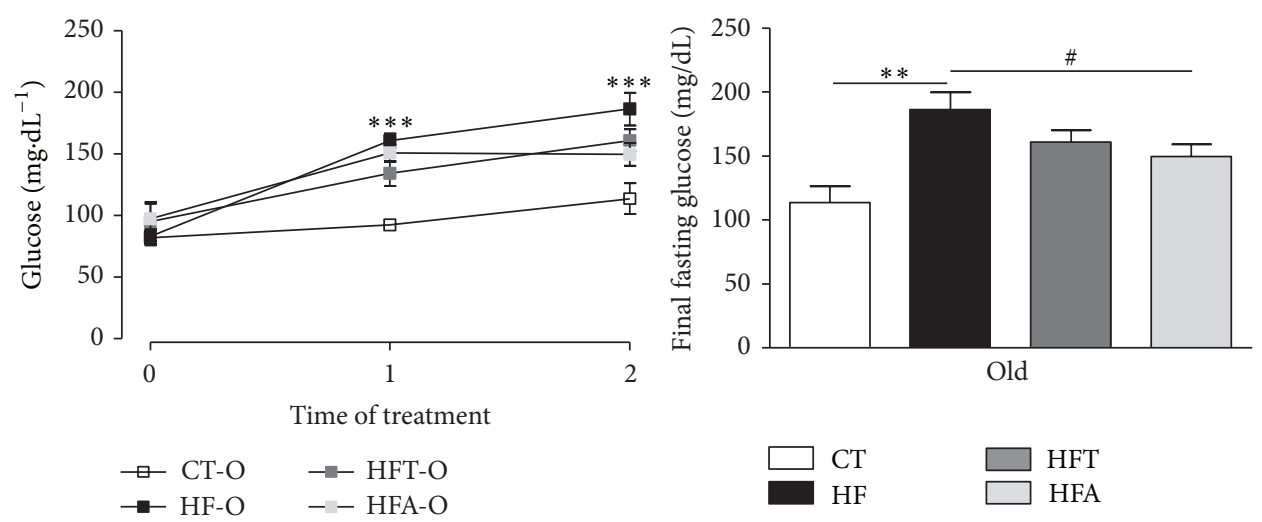

(b)
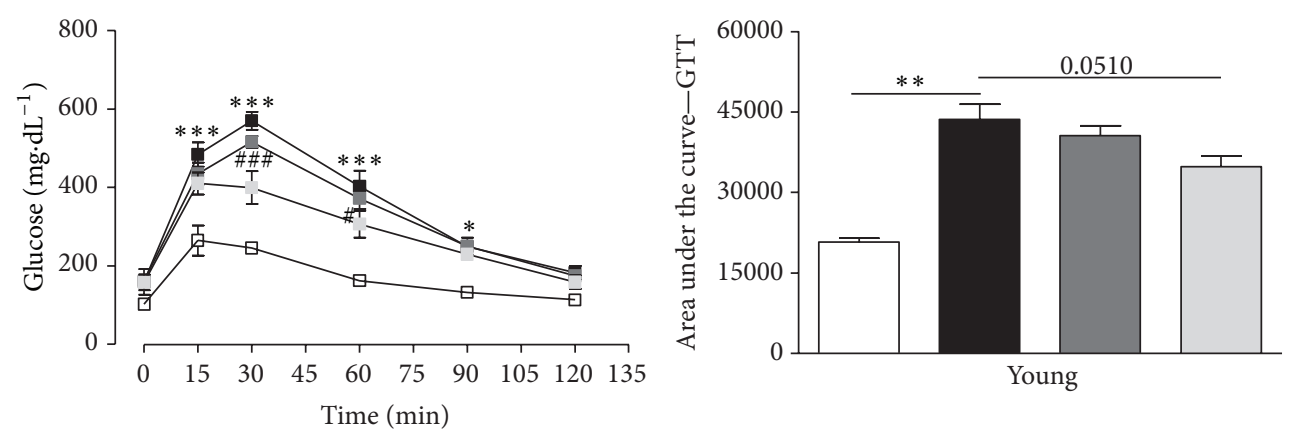

$$
\begin{array}{ll}
\square \text { CT-Y } & -- \text { HFT-Y } \\
\rightarrow \text { HF-Y } & -- \text { HFA-Y }
\end{array}
$$

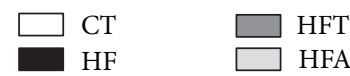

(c)
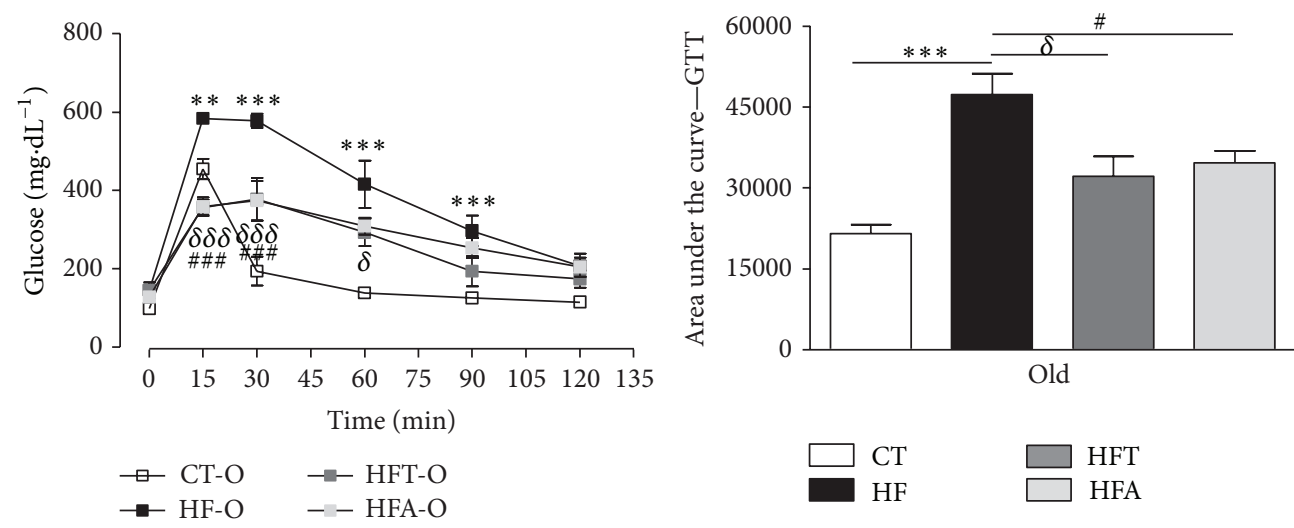

(d)

FIgure 3: Continued. 

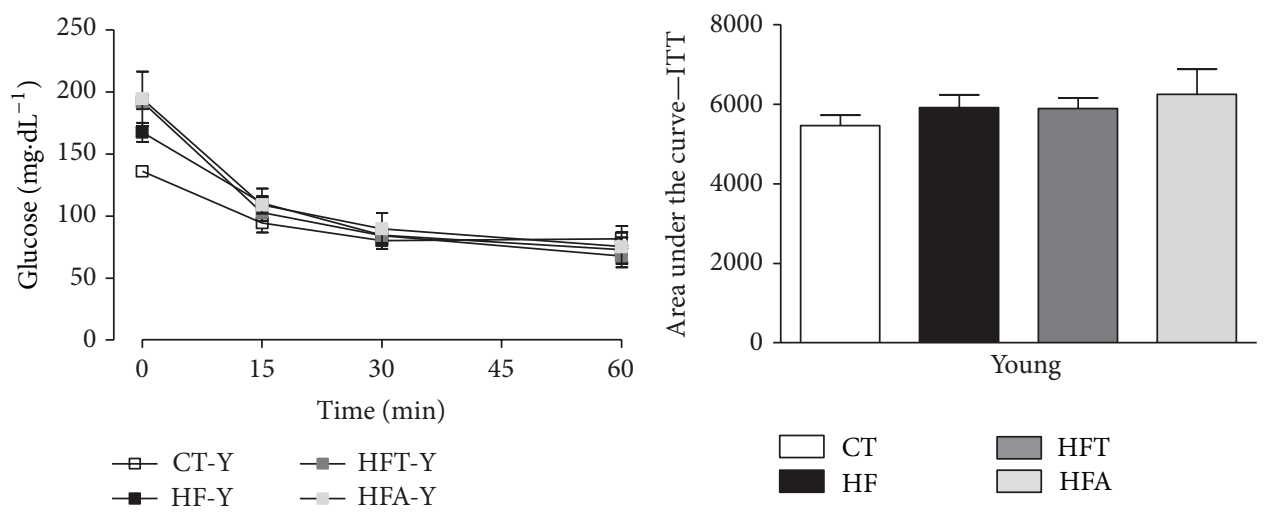

(e)
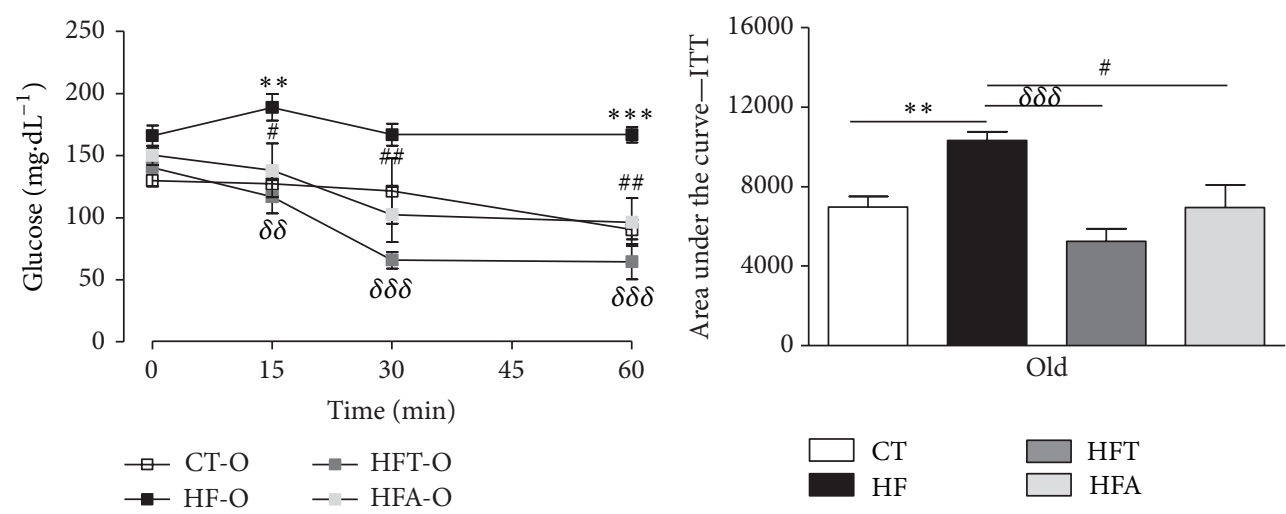

(f)

FIGURE 3: Glycemic profile. Fasting glucose $\left(\mathrm{mg} \cdot \mathrm{dL}^{-1}\right)$ at three different points (basal fasting glucose: Time 0 ; beginning of pharmacological intervention with tempol (50 mg. $\left.\mathrm{kg}^{-1}\right)$ and apocynin $\left(50 \mathrm{mg} \cdot \mathrm{kg}^{-1}\right)$ : Time 1; and the end of pharmacological intervention: Time 2) and end time of fasting glucose in young (a) and old (b) C57BL/6 mice. Evaluation of glucose tolerance and Area under the curve of the test (AUC) in young (c) and old (d) C57BL/6 mice. Evaluation of insulin sensitivity and area under the curve of the test (AUC) in young (e) and old (f) $\mathrm{C} 57 \mathrm{BL} / 6$ mice. $\mathrm{CT}=$ control group; $\mathrm{HF}=$ high fat diet group; HFT $=$ high fat diet + tempol group; HFA = high fat diet + apocynin group. Each point of the graph represents the mean \pm SEM. ANOVA (two-way) with Bonferroni's post hoc test: ${ }^{*} p<0.05 ;{ }^{* *} p<0.01 ;{ }^{* * *} p<0.001$, CT versus HF; ${ }^{\delta \delta} p<0.01 ;{ }^{\delta \delta \delta} p<0.001$, HFT versus HF; ${ }^{\#} p<0.05 ;{ }^{\#} p<0.01 ;{ }^{\# \#} p<0.001$, HFA versus HF. Each bar graph represents the mean \pm SEM. ANOVA (one-way) with Bonferronis post hoc test: ${ }^{*} p<0.05 ;{ }^{* *} p<0.01 ;{ }^{* * *} p<0.001$, CT versus HF; ${ }^{\delta} p<0.05 ;{ }^{\delta \delta \delta} p<0.001$, HFT versus HF; ${ }^{\#} p<0.05$, HFA versus $\mathrm{HF}(n=5-6)$.

Histological assessment from HF-Y and HF-O liver samples presented a pattern of NAFLD, characterized by fatty infiltration (Figures 4(k) and 4(l)). Furthermore, HFD groups (HF-Y and HF-O) showed hepatocellular disorganization and inflammatory zones. Qualitatively, the groups that received tempol (HFT-Y and HFT-O) showed a decrease in hepatic fat accumulation, specifically in macro vesicular steatosis, compared to the respective HFD group. A similar condition was observed for the groups treated with apocynin (HFA-Y and HFA-O) (Figures 4(k) and 4(l)).

The evaluation of the hepatic redox state was based on the quantification of malondialdehyde as a lipid peroxidation measure (Figures 5(a) and 5(b)), as well as the activities of SOD (Figures 5(c) and 5(d)) and CAT (Figures 5(e) and 5(f)) in liver homogenates. Consumption of HFD increased lipid peroxidation, without changes in SOD and CAT activities in liver samples from young (HF-Y) and old (HF-O) mice compared to their respective controls (CT-Y and CT-O). Furthermore, these parameters were not altered after the treatment with tempol and apocynin for both young and old mice.

\section{Discussion}

The major findings of this work are the markedly increased weight gain in aged mice fed a HFD compared to younger ones, as well as the observation that the concomitant intake of HFD and the treatment with antioxidant agents, here represented by apocynin and tempol, improve insulin sensitivity and glucose tolerance in aged mice. Our work also demonstrated that HFD intake induces metabolic alterations similar to those observed in humans presenting with MetS and that NAFLD associated with dietary changes indeed constitute another risk factor for the MetS, the advanced age being an important contributing factor for augmenting such alterations, mainly obesity and hepatic fat deposition [3].

Aging is characterized by a decline in biological functions, with direct influence of lifestyle [34-36]. The data 


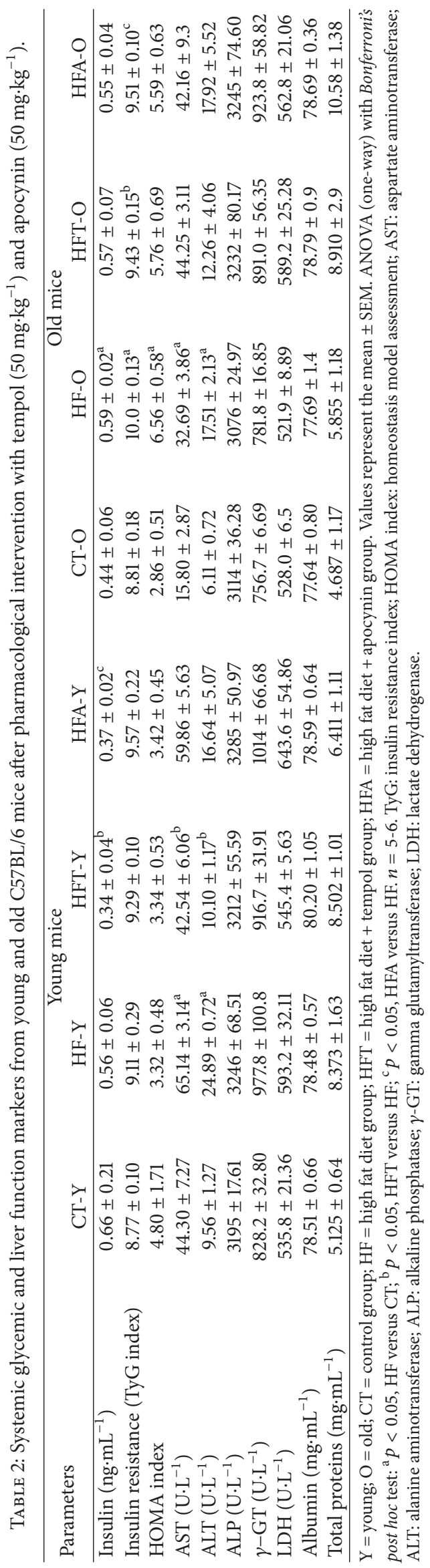



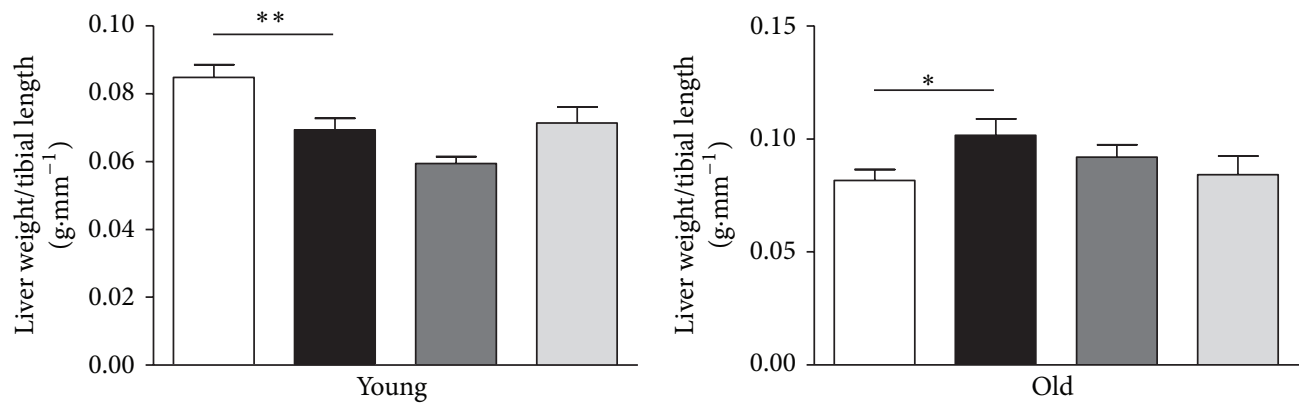

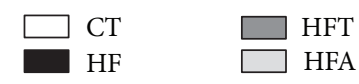

(a)
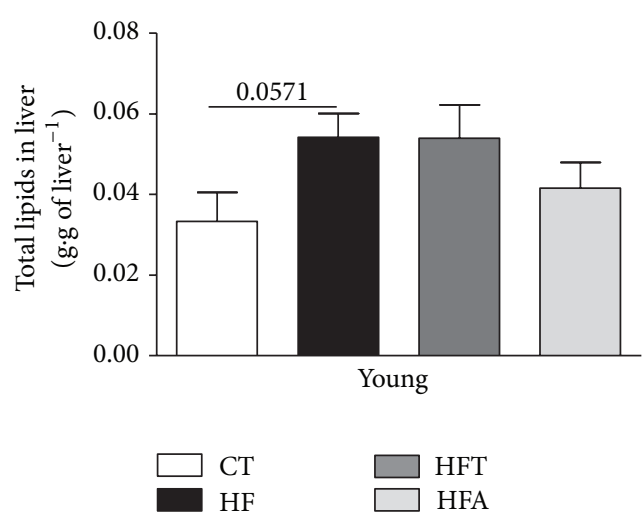

(c)
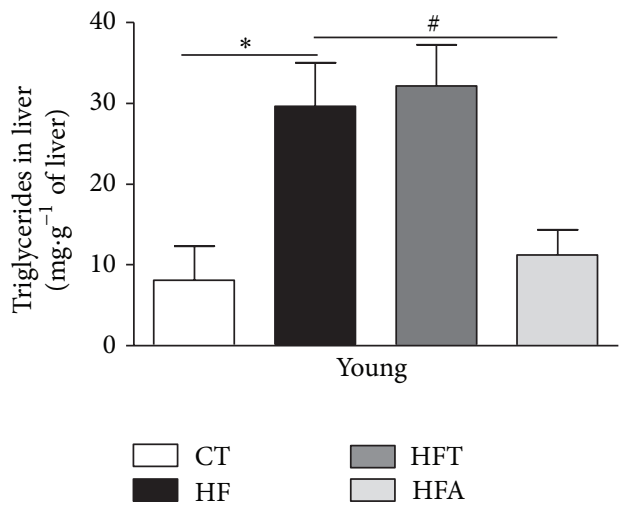

(e)

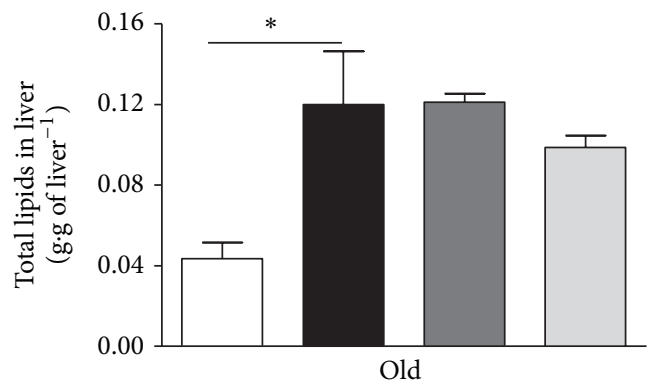

$\square$ CT
HF

(g)

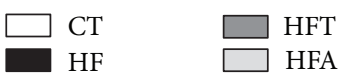

(b)
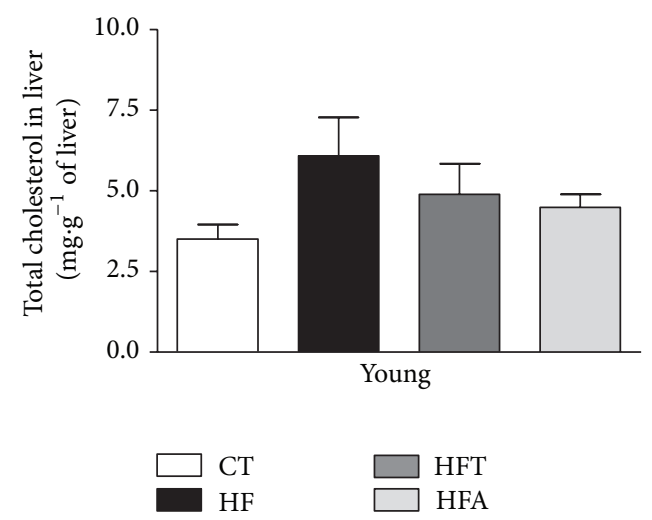

(d)
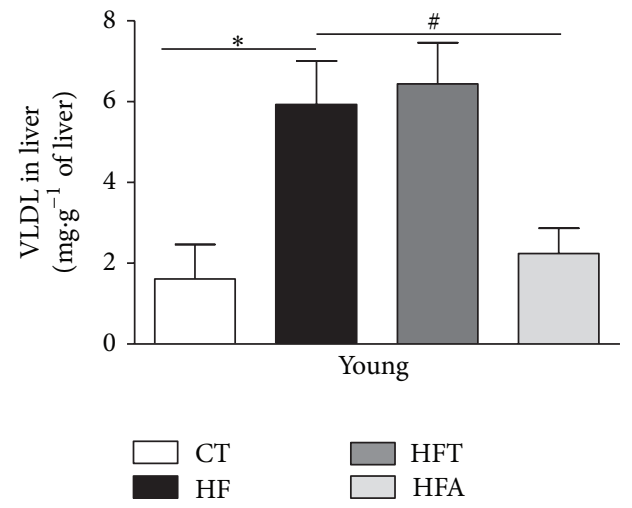

(f)
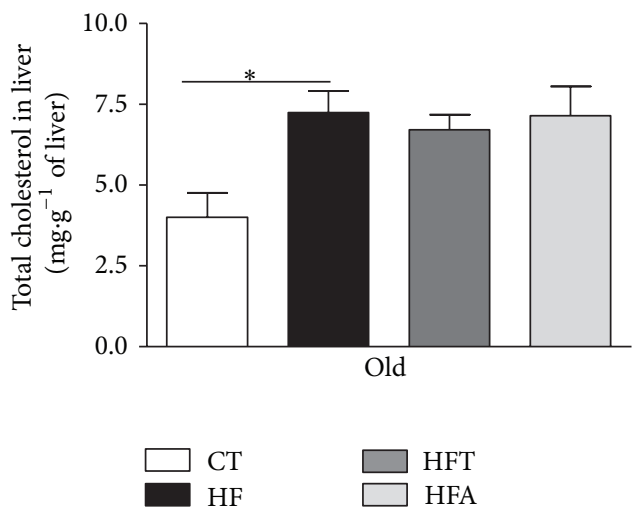

(h)

Figure 4: Continued. 

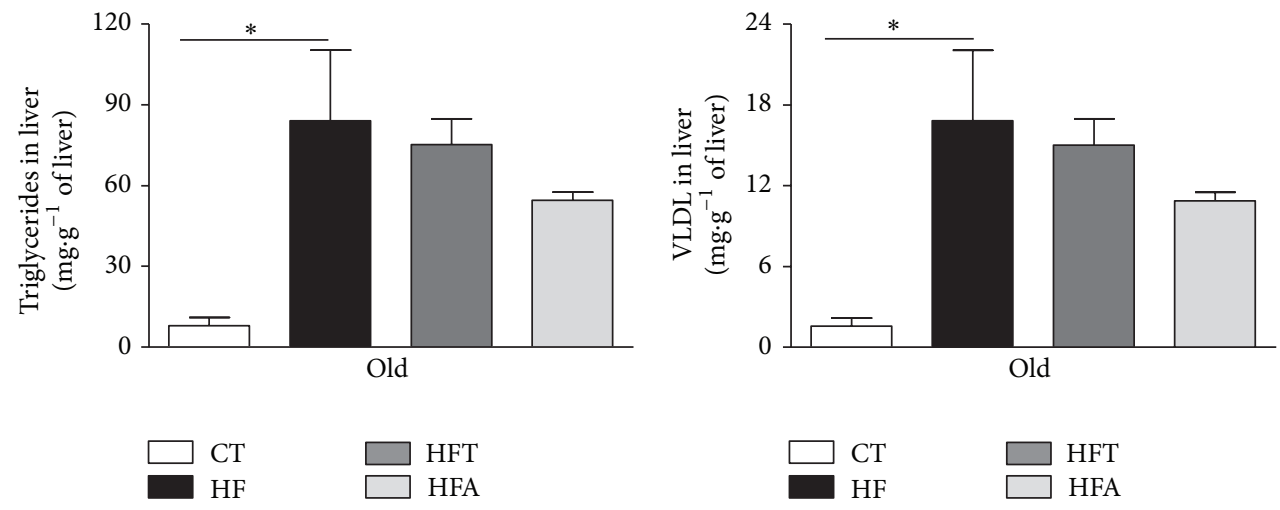

(i)

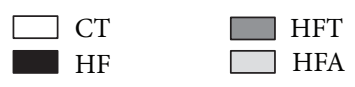

(j)

Young
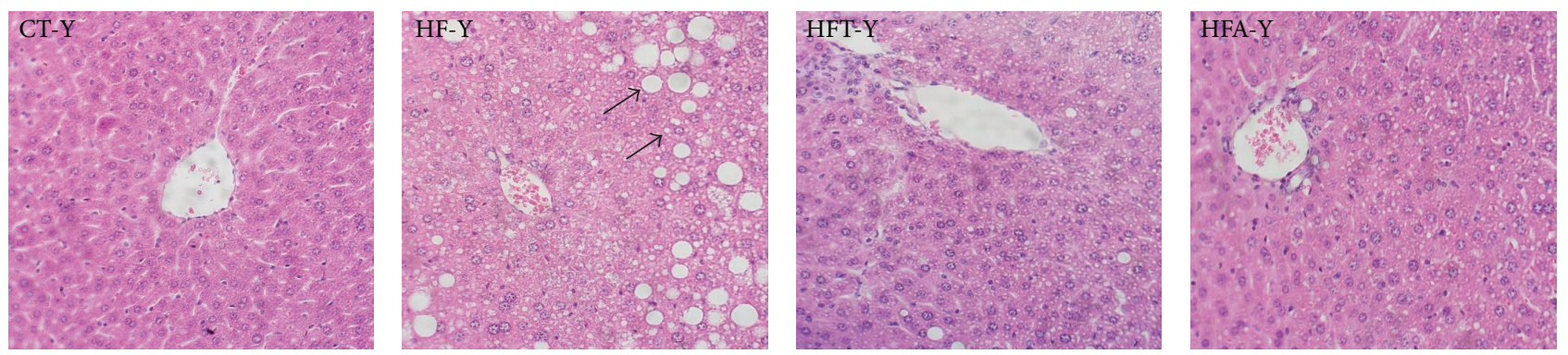

$(\mathrm{k})$

Old
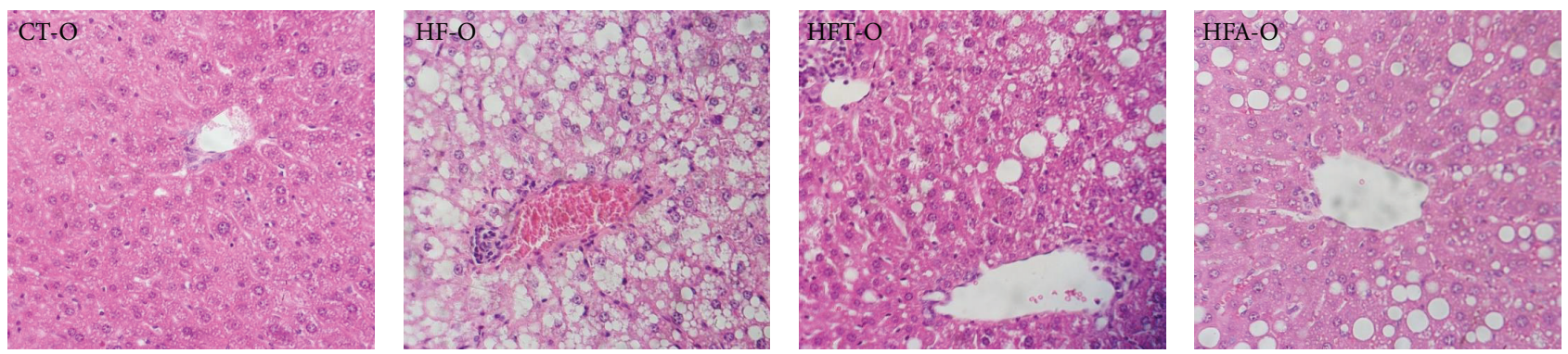

(1)

FIGURE 4: Lipid profile in liver. Liver weight/tibial length $\left(\mathrm{g} \cdot \mathrm{mm}^{-1}\right)$ in young (a) and old (b) C57BL/6 mice. Total lipids in liver ( $\mathrm{g} \cdot \mathrm{g}^{-1}$ of liver) in young (c) and old (g) C57BL/6 mice. Total cholesterol in liver ( $\mathrm{mg}^{-1} \mathrm{~g}^{-1}$ of liver) in young (d) and old (h) C57BL/6 mice. Triglycerides in liver ( $\mathrm{mg}^{-\mathrm{g}^{-1}}$ of liver) in young (e) and old (i) C57BL/6 mice. Very-low-density lipoprotein (VLDL) in liver (mg.g ${ }^{-1}$ of liver) in young (f) and old (j) C57BL/6 mice. Liver histological section from young (k) and old (l) C57BL/6 mice stained with hematoxylin and eosin (400x magnification). The photomicrographs represent the main histological findings in the experimental groups. CT = control group; HF = high fat diet group; HFT = high fat diet + tempol group; HFA = high fat diet + apocynin group. The arrows indicate the fat vesicles (micro and macro). Each bar graph represents the mean \pm SEM. ANOVA (one-way) with Bonferroni's post hoc test: ${ }^{*} p<0.05 ;{ }^{* *} p<0.01$, CT versus HF; ${ }^{*} p<0.05$, HFA versus $\mathrm{HF}(n=5-6)$.

presented herein showed that HFD intake for 14 weeks did not induce body weight gain in young mice (20-22 weeks old at the end of the dietary intervention). Nevertheless, this period was effective in inducing a significant increase in body weight in aged animals. However, young animals presented a greater loss of heart and liver tissue when ingesting HFD, which could compensate the HFD-induced obesity expected in this MetS model. Taken together, these data also evidence that aging and HFD synergistically contribute to accentuating the decline in body capacity to metabolize fat, leading to a greater accumulation of visceral adiposity.

Despite the fact that the HFD-fed young mice did not present significant increase in body weight, other metabolic parameters were shown to be considerably augmented due to the HFD consumption, similar to the observations for the old ones, reflecting, in both groups, the onset of MetS [37-39]. Among these metabolic alterations, increased fasting glucose, impaired glucose tolerance, increased lipid profile in 


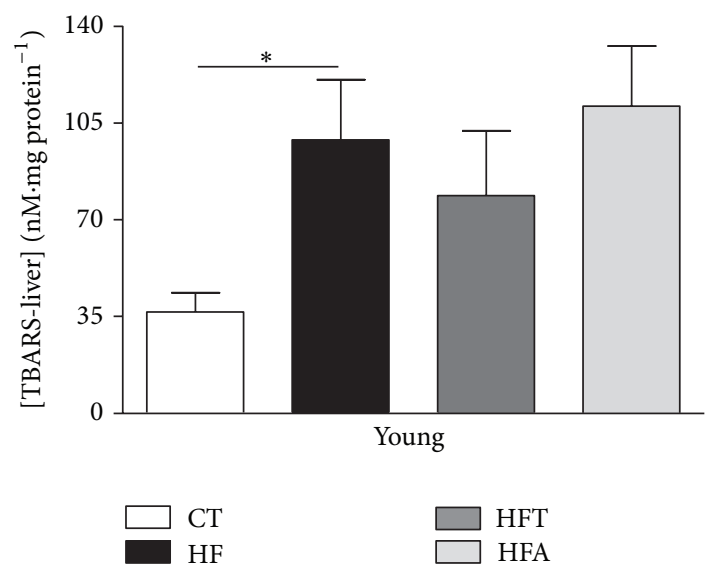

(a)

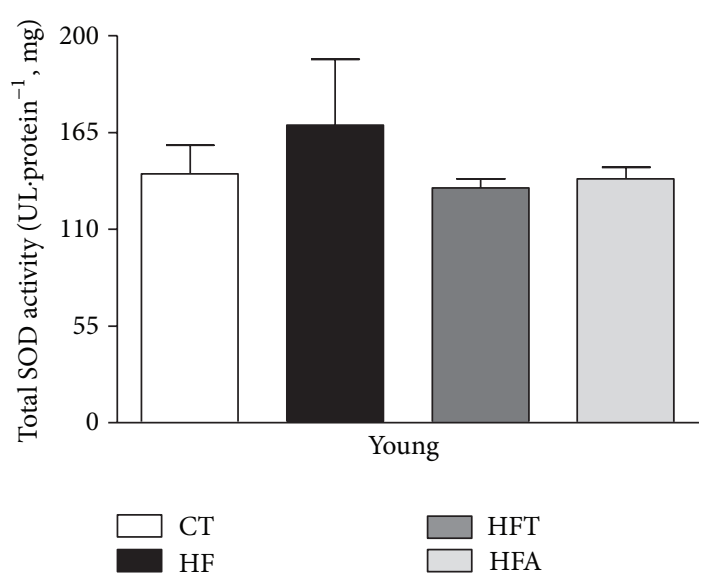

(c)

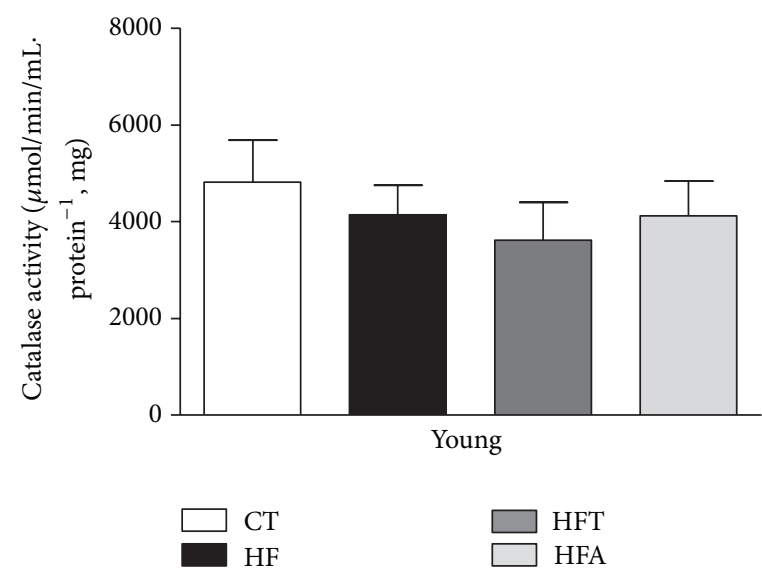

(e)

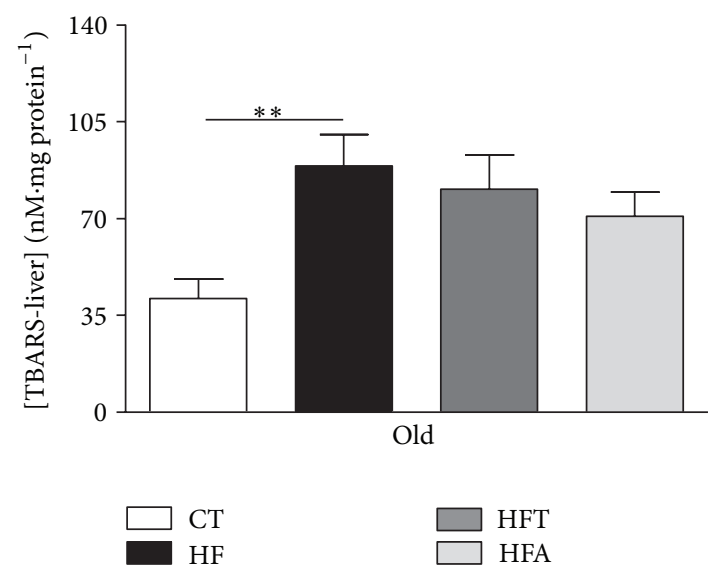

(b)

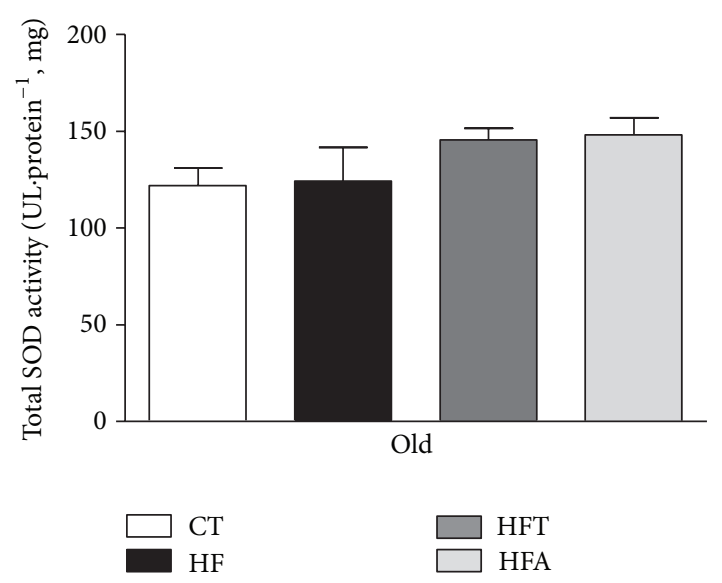

(d)

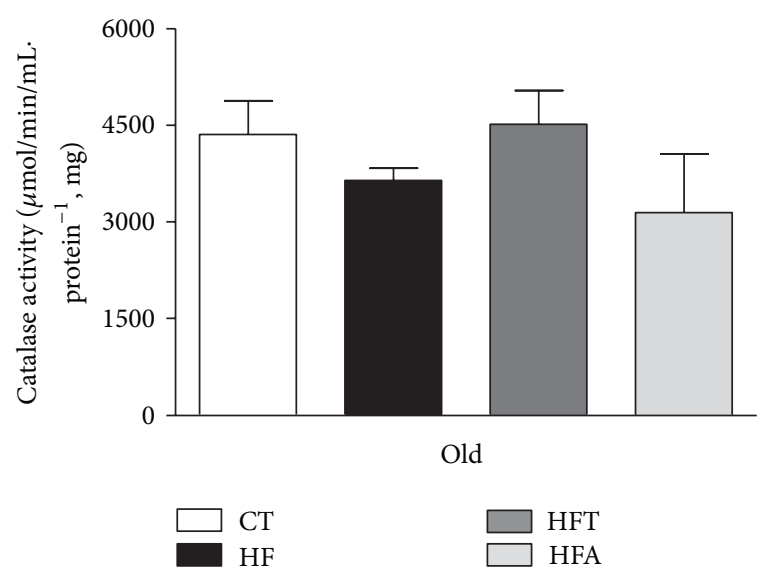

(f)

FIGURE 5: Markers of redox state in liver. Hepatic lipid peroxidation ([TBARS] nM·mg protein ${ }^{-1}$ ) in young (a) and old (b) C57BL/6 mice. Total superoxide dismutase activity (SOD: UI.protein ${ }^{-1}, \mathrm{mg}$ ) in young (c) and old (d) C57BL/6 mice. Catalase activity $\left(\mu \mathrm{mol} / \mathrm{min}^{\mathrm{m}} \mathrm{mL} \cdot \mathrm{protein}{ }^{-1}\right.$, $\mathrm{mg}$ ) in young C57BL/6 (e) and old mice (f). Each bar graph represents the mean \pm SEM. ANOVA (one-way) with Bonferroni's post hoc test: ${ }^{*} p<0.05 ;{ }^{* *} p<0.01, \mathrm{CT}$ versus HF $(n=5-6)$.

plasma, and greater abdominal and hepatic fat deposition, associated with increased AST and ALT plasma levels, could be numbered. However, HFD-induced insulin resistance, augmentation in TyG and HOMA indexes, and the increase in plasma insulin concentration were only observed in aged animals.

It is already known that the main contributing factor in the aging process and the early development of metabolic 
diseases is represented by the oxidative stress [34], with the latter enhancing the metabolic and vascular effects evoked by $\operatorname{HFD}[39,40]$. In this sense, the referred couple of factors, advanced age and dietary changes, might contribute to increase the production of ROS which, in turn, directly contribute to the onset and/or maintenance of glucose intolerance and insulin resistance $[13,41]$. Notwithstanding, after treatment with tempol and apocynin, concomitant with the HFD intake, a significant improvement in these parameters related to glucose metabolism was observed in aged mice, in addition to the decrease in triglycerides and VLDL in plasma. More interestingly, apocynin also decreased these parameters in liver, corroborating the findings of improved insulin sensitivity, as the augmented levels of these markers in the liver are associated with insulin resistance. Together, these data suggest that the increase in $\mathrm{NAD}(\mathrm{P}) \mathrm{H}$ oxidase activity and the concentration of ${ }^{\bullet} \mathrm{O}_{2}{ }^{-}$may be involved in the mechanisms associated with the regulation of the glycemic and lipid profile, with the presence of oxidative stress being possible in this dietary model for MetS.

Tempol acts as a scavenger of ${ }^{\bullet} \mathrm{O}_{2}{ }^{-}[41,42]$. Therefore, it is plausible to assume that the use of this pharmacological agent in the aged group in the current study may have contributed to the reduction of ${ }^{\bullet} \mathrm{O}_{2}{ }^{-}$and, thus, to the improvement in glucose tolerance and insulin sensitivity. In accordance with this statement, Banday and colleagues [41] found that tempol ameliorates insulin sensitivity by promoting a reduction in oxidative stress in obese Zucker rats, this way improving insulin-mediated glucose uptake.

Apocynin, through its antioxidant action and/or its inhibitory effect on the $\mathrm{NAD}(\mathrm{P}) \mathrm{H}$ oxidase enzymatic complex, probably contributed to the observed metabolic improvement at least partially by the reduced participation of $\operatorname{ROS}[13,20]$, ultimately suggesting that the increased activity of the NAD(P)H oxidase enzymatic complex may play an essential role in mechanisms related to the impaired glycemic profile observed in the present work.

In line with these observations, Espinosa and colleagues [16] showed that an increase in NAD(P)H oxidase 2 expression in skeletal muscle appears to be involved in the augmented $\mathrm{H}_{2} \mathrm{O}_{2}$ release upon insulin stimulation in insulinresistant mice [16]. Furthermore, the same study found that, after treatment with apocynin for 8 weeks, HFD-fed mice showed improved glucose tolerance and decreased fasting insulin concentration. In addition, another experimental work showed that the treatment with apocynin for 5 weeks in HFD-fed mice was effective in improving glucose tolerance and insulin sensitivity [9]. These studies, in part, corroborate our findings concerning the antioxidant treatment and glucose metabolism.

Advanced age also favored significant accumulation of abdominal fat, a situation which implies a negative impact on glycemic control [43]. Furthermore, the significant hepatic fat deposition was more pronounced in aged animals, as observed by the intense presence of micro and macro fat vacuoles. Concerning this issue, the relation between age and the degree of obesity and fat deposition was observed, due to the consumption of HFD for 14 weeks. However, after treatment with tempol and apocynin, the qualitative analysis from liver histology showed a less accentuated presence of fat vacuoles, quantitatively confirmed in HFA-O group by a decrease in triglycerides and VLDL. Additionally, tempol reduced the hepatic damage caused by HFD, as observed by the reduction of AST and ALT, both markers of liver injury. These data suggest that the antioxidant activity of apocynin and the scavenger activity of tempol on ${ }^{\circ} \mathrm{O}_{2}{ }^{-}$seem to reduce the hepatic oxidation of fatty acids during the chronic treatment concomitant with the consumption of HFD, preventing liver injury, and that the increased susceptibility of HFDinduced MetS in old mice could be due to the increase of redox imbalance during the aging process. Corroborating the histological findings of the present work, several studies showed the HFD-induced hepatic fat accumulation $[3,40]$.

Still, while assessing the liver histology, the presence of inflammatory agglomerates suggests a direct participation of hepatic fat accumulation in the elevated index of lipid peroxidation observed in this organ, once the inflammatory milieu evoked by fat deposition may contribute to augmenting the local production of ROS. Taken together, these data suggest that HFD induced a state of steatohepatitis, a condition which characterizes the NAFLD [8], and that local oxidative stress may be present and contribute in a direct manner to the progression of NAFLD as well $[6,44]$. Such occurrence could be more likely due to the excessive production of ROS than by a decrease and/or deficiency of antioxidant capacity, since the activity of antioxidant enzymes SOD and CAT did not significantly differ between HFD and chow diet groups.

The products generated in the process of lipid peroxidation act as reactive agents, causing cell damage, which amplifies the oxidative stress effects. Moreover, they induce the production of inflammatory cytokines and subsequent fibrosis, via activation of hepatic stellate cells [45]. Additionally, there exists a correlation between plasma and hepatic oxidative stress and clinical and histological findings in patients presenting with NAFLD [44]. Accordingly, these observations reassert the contribution of oxidative stress in the progression of NAFLD in humans and, under this aspect, our study points to the possible participation of the altered oxidative state, arising from HFD consumption, in the onset of steatohepatitis.

In this context, our results also indicate that the normal hepatic redox state may be altered, possibly by an increase in $\mathrm{NAD}(\mathrm{P}) \mathrm{H}$ oxidase activity and the elevated ${ }^{\circ} \mathrm{O}_{2}{ }^{-}$production. Uchiyama and colleagues [46] suggest that oxidative stress enhances hepatic fat deposition due to the degradation of apolipoprotein B in the liver, indicating that such molecule is a target for ROS. Reinforcing these observations, such degradation process is caused by lipid peroxidation, as stated in a study by Pan and colleagues [25].

In this sense, our data suggest that increased hepatic lipid peroxidation in HFD animals may have directly contributed to inhibiting hepatic release of VLDL to the systemic milieu. In addition, different studies showed apocynin as an effective pharmacological agent in ameliorating hyperlipidemia, hepatic steatosis [2], and insulin resistance [9], besides reducing inflammatory factors such as leptin, interleukin-6, and TNF$\alpha$ [47], thereby indicating a protective role in diseases related to the MetS and in the progression of NAFLD. 
Concerning the effects of apocynin on the oxidative state, Meng and colleagues [9] showed that lipid peroxidation, both hepatic and in plasma, was significantly reduced after an oral treatment with such compound for 5 weeks. Furthermore, the authors observed an increase in SOD activity and a reduction in CAT activity in the liver, after treatment with apocynin. Paradoxically, our findings differ in part from those in the aforementioned study, since only in plasma, but not in liver, the lipid peroxidation was decreased after apocynin treatment in old mice. However, once the advanced age itself represents a factor which culminates with the emergence of redox imbalance [39], it is plausible to suggest that in the liver the difference between treated and untreated groups with antioxidant agents might have been masked by this factor.

In summary, our findings demonstrate that old mice are more susceptible to HFD-induced metabolic changes than young ones, with ROS generation presenting a close relationship with the progression of NAFLD. Furthermore, the improvement in glucose and lipid metabolism-related parameters and the attenuation of the progression of NAFLD in MetS mice, by using antioxidant agents, appears to be due to the ability of these pharmacological agents to inhibit the generation and accumulation of reactive species. Collectively, our results point to the cross talk in the triad HFD-agingoxidative stress, once the HFD-induced metabolic derangements may be more pronounced with the advance of age and also potentially amenable to pharmacological interventions.

\section{Conflict of Interests}

The authors declare there is no conflict of interests regarding the publication of this paper.

\section{Acknowledgments}

The authors wish to thank Maria de Fátima Maia Sarmento and Delma Paes de Souza for their technical assistance during histology preparation and Iris Apostel-Krause for their assistance with the English revision of the paper. The authors wish to thank Coordenação de Aperfeiçoamento de Pessoal de Nível Superior (CAPES; PROCAD-NF 2450/2008) and Conselho Nacional de Desenvolvimento Científico e Tecnológico (CNPq; 483049/2009-3), for the financial support, and Professor Dr. Michael Bader and Dr. Natalia Alenina (Max Delbrück Center for Molecular Medicine, Berlin, Germany) for the donation of some reagents used during the assessment of the oxidative status. Valéria Nunes-Souza, Cheila Juliana César-Gomes, and Lucas José Sá Da Fonseca received a Master's Scholarship from CNPq, FAPEAL, and CAPES, respectively.

\section{References}

[1] A. Kotronen and H. Yki-Järvinen, "Fatty liver: a novel component of the metabolic syndrome," Arteriosclerosis, Thrombosis, and Vascular Biology, vol. 28, no. 1, pp. 27-38, 2008.

[2] S. Furukawa, T. Fujita, M. Shimabukuro et al., "Increased oxidative stress in obesity and its impact on metabolic syndrome," The
Journal of Clinical Investigation, vol. 114, no. 12, pp. 1752-1761, 2004.

[3] J. C. Fraulob, R. Ogg-Diamantino, C. Fernandes-Santos, M. B. Aguila, and C. A. Mandarim-de-Lacerda, "A mouse model of metabolic syndrome: insulin resistance, fatty liver and NonAlcoholic Fatty Pancreas Disease (NAFPD) in C57BL/6 mice fed a high fat diet," Journal of Clinical Biochemistry and Nutrition, vol. 46, no. 3, pp. 212-223, 2010.

[4] M. den Boer, P. J. Voshol, F. Kuipers, L. M. Havekes, and J. A. Romijn, "Hepatic steatosis: a mediator of the metabolic syndrome. Lessons from animal models," Arteriosclerosis, Thrombosis, and Vascular Biology, vol. 24, no. 4, pp. 644-649, 2004.

[5] G. C. Farrell and C. Z. Larter, "Nonalcoholic fatty liver disease: from steatosis to cirrhosis," Hepatology, vol. 43, no. 2, pp. S99S112, 2006.

[6] M. Basaranoglu, G. Basaranoglu, and H. Sentürk, "From fatty liver to fibrosis: a tale of 'second hit", World Journal of Gastroenterology, vol. 19, no. 8, pp. 1158-1165, 2013.

[7] W. Dröge, "Free radicals in the physiological control of cell function," Physiological Reviews, vol. 82, no. 1, pp. 47-95, 2002.

[8] K. Qureshi and G. A. Abrams, "Metabolic liver disease of obesity and role of adipose tissue in the pathogenesis of nonalcoholic fatty liver disease," World Journal of Gastroenterology, vol. 13, no. 26, pp. 3540-3553, 2007.

[9] R. Meng, D.-L. Zhu, Y. Bi, D.-H. Yang, and Y.-P. Wang, "Antioxidative effect of apocynin on insulin resistance in high-fat diet mice," Annals of Clinical \& Laboratory Science, vol. 41, no. 3, pp. 236-243, 2011.

[10] J. Raval, S. Lyman, T. Nitta et al., "Basal reactive oxygen species determine the susceptibility to apoptosis in cirrhotic hepatocytes," Free Radical Biology and Medicine, vol. 41, no. 11, pp. 1645-1654, 2006.

[11] R. B. Dorman, C. Wunder, H. Saba, J. L. Shoemaker, L. A. MacMillan-Crow, and R. W. Brock, "NAD $(\mathrm{P}) \mathrm{H}$ oxidase contributes to the progression of remote hepatic parenchymal injury and endothelial dysfunction, but not microvascular perfusion deficits," American Journal of PhysiologyGastrointestinal and Liver Physiology, vol. 290, no. 5, pp. G1025G1032, 2006.

[12] S. Gupte, N. Labinskyy, R. Gupte, A. Csiszar, Z. Ungvari, and J. G. Edwards, "Role of $\mathrm{NAD}(\mathrm{P}) \mathrm{H}$ oxidase in superoxide generation and endothelial dysfunction in Goto-Kakizaki (GK) rats as a model of nonobese NIDDM," PLoS ONE, vol. 5, no. 7, Article ID el1800, 2010.

[13] T. Sonta, T. Inoguchi, H. Tsubouchi et al., "Evidence for contribution of vascular NAD $(\mathrm{P}) \mathrm{H}$ oxidase to increased oxidative stress in animal models of diabetes and obesity," Free Radical Biology and Medicine, vol. 37, no. 1, pp. 115-123, 2004.

[14] H. E. Lob, D. Schultz, P. J. Marvar, R. L. Davisson, and D. G. Harrison, "Role of the NADPH oxidases in the subfornical organ in angiotensin II-induced hypertension," Hypertension, vol. 61, no. 2, pp. 382-387, 2013.

[15] D. W. Trott, J. W. Seawright, M. J. Luttrell, and C. R. Woodman, "NAD $(\mathrm{P}) \mathrm{H}$ oxidase-derived reactive oxygen species contribute to age-related impairments of endothelium-dependent dilation in rat soleus feed arteries," Journal of Applied Physiology, vol. 110, no. 5, pp. 1171-1180, 2011.

[16] A. Espinosa, C. Campos, A. Díaz-Vegas et al., "Insulindependent $\mathrm{H}_{2} \mathrm{O}_{2}$ production is higher in muscle fibers of mice 
fed with a high-fat diet," International Journal of Molecular Sciences, vol. 14, no. 8, pp. 15740-15754, 2013.

[17] L. A. Rabêlo, V. N. de Souza, L. J. S. da Fonseca, and W. O. Sampaio, "Redox unbalance: NADPH oxidase as therapeutic target in blood pressure control," Arquivos Brasileiros de Cardiologia, vol. 94, no. 5, pp. 643-693, 2010.

[18] A. B. Salmon, A. Richardson, and V. I. Pérez, "Update on the oxidative stress theory of aging: does oxidative stress play a role in aging or healthy aging?" Free Radical Biology and Medicine, vol. 48 , no. 5 , pp. 642-655, 2010.

[19] V. I. Pérez, A. Bokov, H. V. Remmen et al., "Is the oxidative stress theory of aging dead?" Biochimica et Biophysica Acta-General Subjects, vol. 1790, no. 10, pp. 1005-1014, 2009.

[20] L.-S. Lu, C.-C. Wu, L.-M. Hung et al., "Apocynin alleviated hepatic oxidative burden and reduced liver injury in hypercholesterolaemia," Liver International, vol. 27, no. 4, pp. 529-537, 2007.

[21] S. Polakof, M. E. Díaz-Rubio, D. Dardevet et al., "Resistant starch intake partly restores metabolic and inflammatory alterations in the liver of high-fat-diet-fed rats," Journal of Nutritional Biochemistry, vol. 24, no. 11, pp. 1920-1930, 2013.

[22] K. Lim, S. L. Burke, and G. A. Head, "Obesity-related hypertension and the role of insulin and leptin in high-fat-fed rabbits," Hypertension, vol. 61, no. 3, pp. 628-634, 2013.

[23] P. G. Reeves, F. H. Nielsen, and G. C. Fahey Jr., "AIN-93 purified diets for laboratory rodents: final report of the American Institute of Nutrition ad hoc writing committee on the reformulation of the AIN-76A rodent diet," Journal of Nutrition, vol. 123, no. 11, pp. 1939-1951, 1993.

[24] P. G. Reeves, K. L. Rossow, and J. Lindlauf, "Development and testing of the AIN-93 purified diets for rodents: results on growth, kidney calcification and bone mineralization in rats and mice," The Journal of Nutrition, vol. 123, no. 11, pp. 1923-1931, 1993.

[25] M. Pan, A. I. Cederbaum, Y.-L. Zhang, H. N. Ginsberg, K. J. Williams, and E. A. Fisher, "Lipid peroxidation and oxidant stress regulate hepatic apolipoprotein B degradation and VLDL production," The Journal of Clinical Investigation, vol. 113, no. 9, pp. 1277-1287, 2004.

[26] W. T. Friedewald, R. I. Levy, and D. S. Fredrickson, "Estimation of the concentration of low-density lipoprotein cholesterol in plasma, without use of the preparative ultracentrifuge," Clinical Chemistry, vol. 18, no. 6, pp. 499-502, 1972.

[27] L. E. Simental-Mendía, M. Rodríguez-Morán, and F. GuerreroRomero, "The product of fasting glucose and triglycerides as surrogate for identifying insulin resistance in apparently healthy subjects," Metabolic Syndrome and Related Disorders, vol. 6, no. 4, pp. 299-304, 2008.

[28] F. Guerrero-Romero, L. E. Simental-Mendía, M. GonzálezOrtiz et al., "The product of triglycerides and glucose, a simple measure of insulin sensitivity. Comparison with the euglycemic-hyperinsulinemic clamp," Journal of Clinical Endocrinology and Metabolism, vol. 95, no. 7, pp. 3347-3351, 2010.

[29] D. R. Matthews, J. P. Hosker, A. S. Rudenski, B. A. Naylor, D. F. Treacher, and R. C. Turner, "Homeostasis model assessment: insulin resistance and $\beta$-cell function from fasting plasma glucose and insulin concentrations in man," Diabetologia, vol. 28, no. 7, pp. 412-419, 1985.
[30] M. M. Bradford, "A rapid and sensitive method for the quantitation of microgram quantities of protein utilizing the principle of protein-dye binding," Analytical Biochemistry, vol. 72, no. 1-2, pp. 248-254, 1976.

[31] J. Folch, M. Lees, and G. H. Sloane Stanley, "A simple method for the isolation and purification of total lipides from animal tissues," The Journal of Biological Chemistry, vol. 226, no. 1, pp. 497-509, 1957.

[32] H. Ohkawa, N. Ohishi, and K. Yagi, "Assay for lipid peroxides in animal tissues by thiobarbituric acid reaction," Analytical Biochemistry, vol. 95, no. 2, pp. 351-358, 1979.

[33] P. Xu, A. C. Costa-Goncalves, M. Todiras et al., "Endothelial dysfunction and elevated blood pressure in MAS gene-deleted mice," Hypertension, vol. 51, no. 2, pp. 574-580, 2008.

[34] R. S. Sohal and R. Weindruch, "Oxidative stress, caloric restriction, and aging," Science, vol. 273, no. 5271, pp. 59-63, 1996.

[35] S. Ikeyama, G. Kokkonen, S. Shack, X.-T. Wang, and N. J. Holbrook, "Loss in oxidative stress tolerance with aging linked to reduced extracellular signal-regulated kinase and Akt kinase activities," The FASEB Journal, vol. 16, no. 1, pp. 114-116, 2002.

[36] J. Wanagat, D. B. Allison, and R. Weindruch, "Caloric intake and aging: mechanisms in rodents and a study in nonhuman primates," Toxicological Sciences, vol. 52, no. 2, pp. 35-40, 1999.

[37] J. Molnar, S. Yu, N. Mzhavia, C. Pau, I. Chereshnev, and H. M. Dansky, "Diabetes induces endothelial dysfunction but does not increase neointimal formation in high-fat diet fed C57BL/6J mice," Circulation Research, vol. 96, no. 11, pp. 1178-1184, 2005.

[38] H. Sone and Y. Kagawa, "Pancreatic beta cell senescence contributes to the pathogenesis of type 2 diabetes in high-fat dietinduced diabetic mice," Diabetologia, vol. 48, no. 1, pp. 58-67, 2005.

[39] A. R. Collins, C. J. Lyon, X. Xia et al., "Age-accelerated atherosclerosis correlates with failure to upregulate antioxidant genes," Circulation Research, vol. 104, no. 6, pp. e42-e54, 2009.

[40] A. A. Gupte, J. Z. Liu, Y. Ren et al., "Rosiglitazone attenuates ageand diet-associated nonalcoholic steatohepatitis in male lowdensity lipoprotein receptor knockout mice," Hepatology, vol. 52, no. 6, pp. 2001-2011, 2010.

[41] A. A. Banday, A. Marwaha, L. S. Tallam, and M. F. Lokhandwala, "Tempol reduces oxidative stress, improves insulin sensitivity, decreases renal dopamine D1 receptor hyperphosphorylation, and restores D1 receptor-G-protein coupling and function in obese Zucker rats," Diabetes, vol. 54, no. 7, pp. 2219-2226, 2005.

[42] C. S. Wilcox, "Effects of tempol and redox-cycling nitroxides in models of oxidative stress," Pharmacology and Therapeutics, vol. 126, no. 2, pp. 119-145, 2010.

[43] A. Gastaldelli, Y. Miyazaki, M. Pettiti et al., "Metabolic effects of visceral fat accumulation in type 2 diabetes," Journal of Clinical Endocrinology and Metabolism, vol. 87, no. 11, pp. 5098-5103, 2002.

[44] L. A. Videla, R. Rodrigo, M. Orellana et al., "Oxidative stressrelated parameters in the liver of non-alcoholic fatty liver disease patients," Clinical Science, vol. 106, no. 3, pp. 261-268, 2004.

[45] J. D. Browning and J. D. Horton, "Molecular mediators of hepatic steatosis and liver injury," The Journal of Clinical Investigation, vol. 114, no. 2, pp. 147-152, 2004. 
[46] S. Uchiyama, T. Shimizu, and T. Shirasawa, "CuZn-SOD deficiency causes $A p o B$ degradation and induces hepatic lipid accumulation by impaired lipoprotein secretion in mice," The Journal of Biological Chemistry, vol. 281, no. 42, pp. 31713-31719, 2006.

[47] R. Meng, D.-L. Zhu, Y. Bi, D.-H. Yang, and Y.-P. Wang, "Apocynin improves insulin resistance through suppressing inflammation in high-fat diet-induced obese mice," Mediators of Inflammation, vol. 2010, Article ID 858735, 9 pages, 2010. 


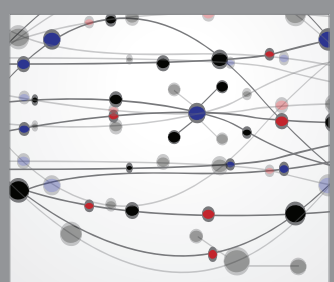

The Scientific World Journal
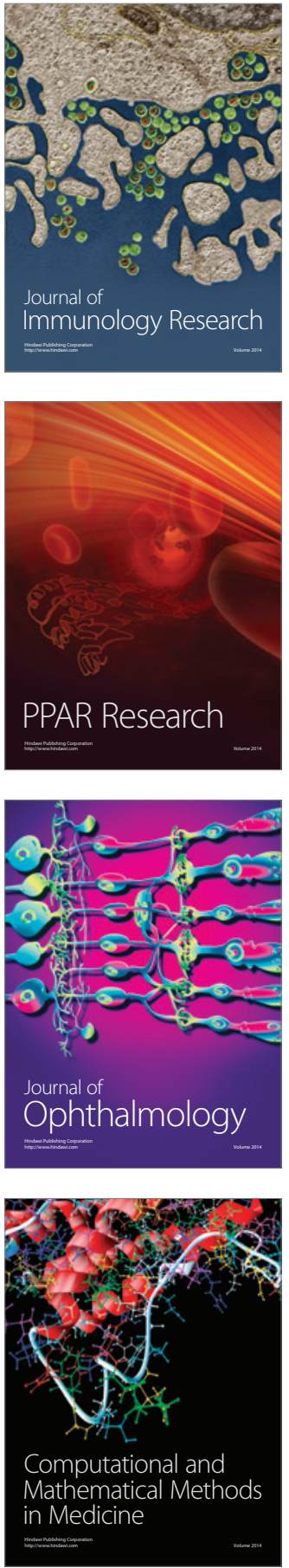

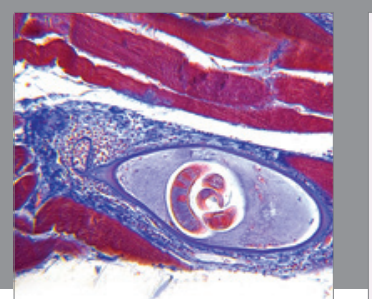

Gastroenterology Research and Practice

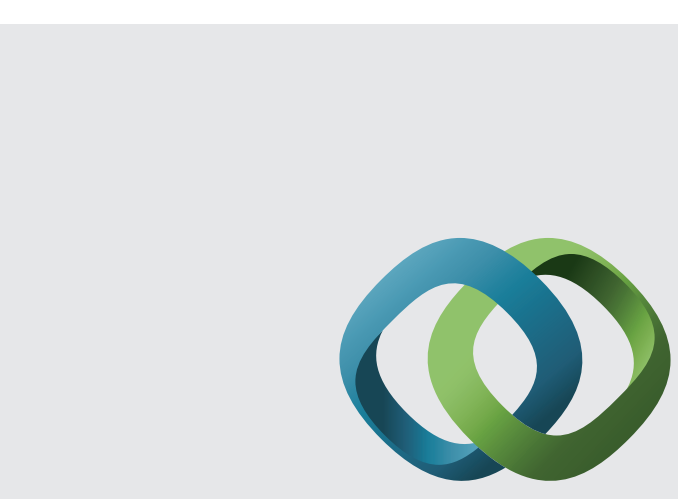

\section{Hindawi}

Submit your manuscripts at

http://www.hindawi.com
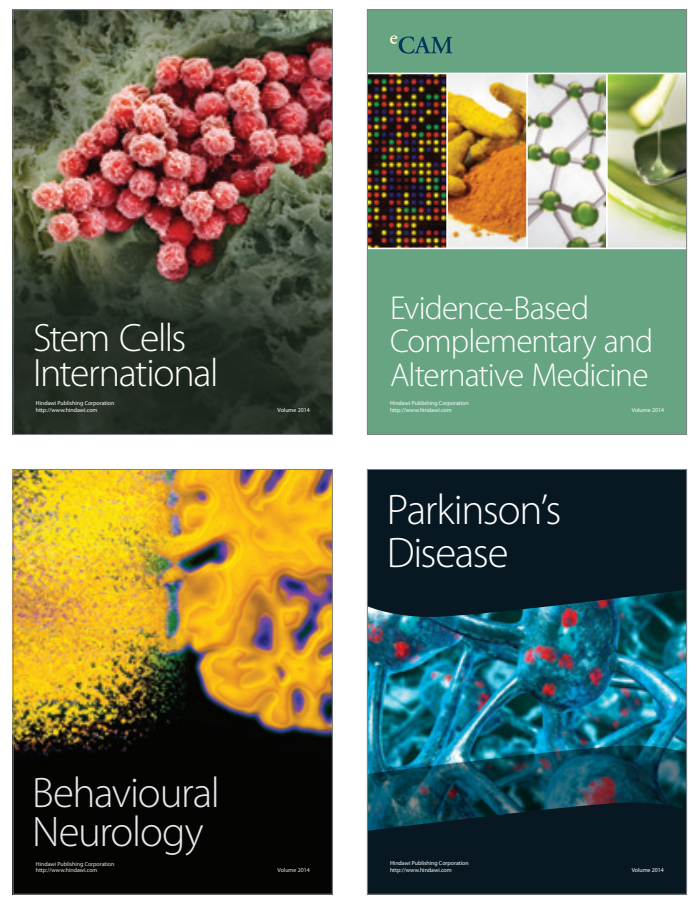
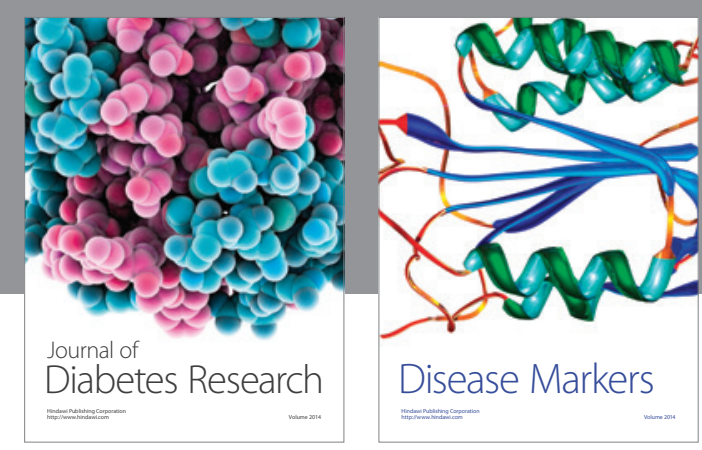

Disease Markers
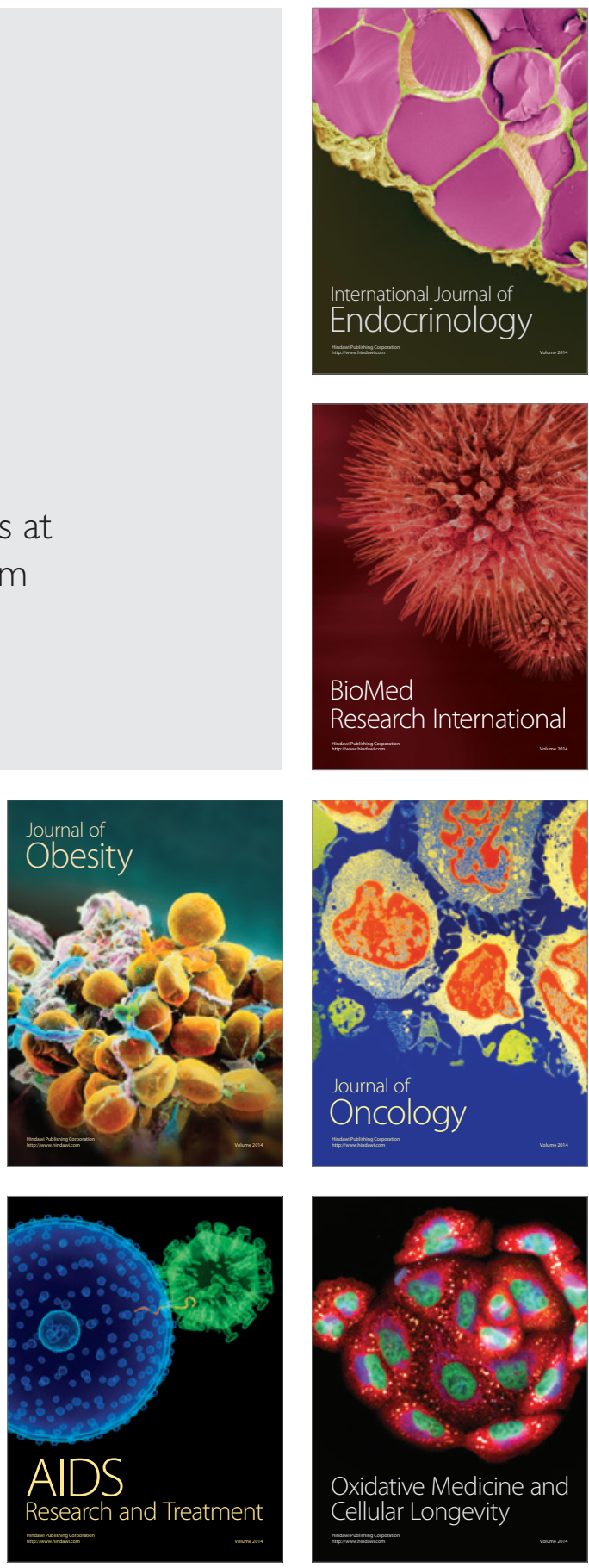